\title{
Analyse systématique des mobilités d'un mécanisme
}

\author{
Abdelfattah Mlika ${ }^{1,4, a}$, Habib Bettaieb $^{2,4}$ et Abdelwaheb Dogui ${ }^{3,4}$ \\ 1 Institut préparatoire aux études d'ingénieurs de Monastir, Avenue Ibn El Jazzar, 5019 Monastir, Tunisie \\ 2 École préparatoire aux académies militaires, Rue Maréchal Tito, 4029 Sousse, Tunisie \\ 3 École nationale d’ingénieurs de Monastir, Avenue Ibn El Jazzar, 5019 Monastir, Tunisie \\ 4 Laboratoire de Génie Mécanique, École nationale d'ingénieurs de Monastir, Rue Ibn El Jazzar, 5019 Monastir, Tunisie
}

Reçu le 3 septembre 2002, accepté le 30 janvier 2004

\begin{abstract}
Résumé - Dans cet article nous proposons une méthode d'analyse cinématique systématique d'un mécanisme dans une configuration donnée. À partir de la matrice de connexion associée au mécanisme et en utilisant la technique de concaténation, on détermine en premier un ensemble de chemins les plus courts reliant le bâti à chaque liaison. À partir de cet ensemble, on détermine systématiquement un jeu de cycles indépendants. La mise en équations automatique est réalisée simultanément avec la détection des cycles par une technique d'assemblage des matrices élémentaires associées aux liaisons. Le traitement du système linéaire obtenu permet de dégager tous les jeux possibles des paramètres cinématiques indépendants dont une méthode originale de présentation permet le choix et la localisation rapide.
\end{abstract}

Mots clés : Cycles indépendants / concaténation / paramètres cinématiques indépendants / analyse des mobilités / technique d'assemblage

\begin{abstract}
Systematic mobility analysis of mechanism. In this paper, we present a systematic method of mobility analysis of mechanisms. This method allows the determination of a set of independent loops using the connectivity matrix associated with the mechanism. The concatenation technique was used in this case. The kinematic equations were built automatically by assembling the elementary matrices associated with each joint. The analysis of the obtained linear system yields all possible sets of kinematic independent parameters. An original method of presentation of these sets allows the user to identify easily any one of the sets of independent kinematic parameters.
\end{abstract}

Key words: Independent loops / independant kinematic parameters / mobility analysis / assembling technic

\section{Introduction}

La recherche d'une solution optimale pour un mécanisme doit commencer dès la phase d'avant projet. À ce stade, les informations utiles pour un concepteur sont, pour l'essentiel, les degrés de mobilité et d'hyperstaticité et les relations entrée-sortie. Depuis quelque temps, les recherches se sont penchées sur le développement des méthodes systématiques capables de fournir d'autres informations qui peuvent assister le concepteur dans sa tâche $[1,2]$. Parmi ces informations, on distingue les libertés des liaisons rendues bloquées après insertion dans le mécanisme, les solutions isostatiques et la distribution ou la répartition des mobilités $[3,4]$. Ce dernier point, semble apporter une aide précieuse dans une

\footnotetext{
a Auteur correspondant : Abdel.Mlika@ipeim.rnu.tn
}

phase de conception ou d'exploration d'un mécanisme. En effet, offrir toutes les possibilités de choix des paramètres cinématiques indépendants correspond à un diagnostic détaillé qui peut déboucher sur des idées inaperçues au départ, ou démasquer une mobilité interne non évidente [5]. Dans ce cadre, le travail de Fayet et Bonnet [3] consiste en premier lieu à déterminer la liaison équivalente ou espace des torseurs cinématiques entre deux solides quelconques dans un mécanisme multicycles. Cela a permis aux auteurs de construire une matrice génératrice $G^{*}$ dont chaque colonne représente la distribution d'une mobilité sur toutes les liaisons. Cette matrice fournit également tous les jeux de paramètres cinématiques indépendants. Toutefois, un calcul intermédiaire du déterminant d'une matrice carré, ayant pour dimension le degré de mobilité, est quasi-nécessaire. Les éléments de cette matrice sont calculés à partir du 


\section{Nomenclature}

\begin{tabular}{|ll|}
\hline $\mathrm{C}_{\mathrm{i}}$ & chemin le plus court associé à la liaison $\ell_{\mathrm{i}}$ \\
$\mathrm{C}$ & ensemble de $\ell$ chemins $\mathrm{C}_{\mathrm{i}}$ \\
$\mathrm{E}_{\mathrm{k}}$ & sous-ensemble des inconnues cinématiques \\
$\mathrm{h}$ & degré d'hyperstaticité \\
$\mathrm{Ic}$ & nombre des inconnues cinématiques \\
$\mathrm{J}(\mathbf{u})$ & sous-ensemble des jeux de paramètres cinématiques indépendants contenant l'inconnue $\mathbf{u}$ \\
$\mathrm{m}$ & degré de mobilité \\
{$[\mathbf{M}]$} & matrice de connexion associée au graphe de liaisons \\
$\mathrm{n}_{\mathrm{tk}}$ & nombre des inconnues à choisir arbitrairement dans un sous-ensemble $\mathrm{E}_{\mathrm{k}}$ \\
$\mathrm{N}_{\mathrm{i}}$ & nombre entier associé à un jeu de paramètres cinématiques indépendants \\
{$[\mathbf{T c}(\mathbf{j} / \mathbf{i})]$} & matrice rectangulaire définissant le torseur $\left\{\vartheta_{\mathrm{j} / \mathrm{i}}\right\}$ au centre du repère global \\
$\mathbf{u}, \mathbf{v}, \mathbf{w}$ & base du repère local d'une liaison \\
$\mathbf{u}_{\mathrm{ji}}, \mathbf{v}_{\mathrm{ji}}, \mathbf{w}_{\mathrm{ji}}$ & composantes dans la base $\mathbf{u}, \mathbf{v}, \mathbf{w}$ du vecteur vitesse de translation de la pièce $\mathrm{j}$ par rapport à la pièce $\mathrm{i}$ \\
$\left\{\mathbf{W}_{\mathrm{i}}\right\}$ & jeu de paramètres cinématiques indépendants \\
$\ell$ & nombre total des liaisons dans un mécanisme \\
$\ell_{\mathrm{i}}$ & liaison n ${ }^{\circ} \mathrm{i}$ d'un mécanisme \\
{$\left[\mathbf{M}_{\mathrm{bâti}}^{x}\right]$} & matrice des chemins de longueur $x$ partant du bâti \\
$\left\{\vartheta_{\mathrm{j} / \mathrm{i}}\right\}$ & torseur cinématique des mouvements de la pièce j par rapport à la pièce i \\
$\alpha_{\mathrm{ji}}, \beta_{\mathrm{ji}}, \gamma_{\mathrm{ji}}$ & composantes dans la base $\mathbf{u}, \mathbf{v}, \mathbf{w}$ du vecteur vitesse de rotation de la pièce $\mathrm{j}$ par rapport à la pièce i \\
\hline
\end{tabular}

produit scalaire des éléments correspondants dans la matrice $\mathrm{G}^{*}$ et de ceux de la base des libertés cinématiques. Cependant, le processus de détermination de la matrice $\mathrm{G}^{*}$ est assez compliqué et se base complètement sur le graphe de liaisons du mécanisme.

Dans ce travail, nous nous intéressons au même problème de détermination des jeux de paramètres cinématiques indépendants. Notre objectif est le développement d'une méthode systématique plus complète pour l'analyse cinématique d'un mécanisme à partir des seules données en liaisons. Notre contribution a concerné la détermination d'un jeu de cycles indépendants, l'identification et la présentation de tous les jeux de paramètres cinématiques indépendants.

Pour la détermination systématique d'un jeu de cycles indépendants, on adopte généralement une procédure à deux étapes. La première étape consiste en une recherche de tous les cycles contenus dans le graphe de liaisons. La seconde s'occupe de la sélection d'un jeu de cycles indépendants parmi l'ensemble des cycles obtenus. L'idée énoncée par Deplanche et Leroy [6] consiste à écrire les équations de fermeture à partir des équations cinématiques d'une base de $\ell$ chemins particuliers ( $\ell$ étant le nombre de liaisons) extraits du graphe. Les auteurs déterminent ces chemins intuitivement et les introduisent comme données pour la suite du processus. Dans ce travail nous avons adapté leur idée à la recherche d'un jeu de cycles indépendants tout en rendant la détermination des $\ell$ chemins complètement automatique à partir des seules données en liaisons.

L'identification des jeux de paramètres cinématiques indépendants se fait à partir de l'analyse du système linéaire obtenu. Cependant, le nombre souvent excessif de ces jeux nous a amené à développer une méthode originale qui permet d'en choisir rapidement un.

\section{Recherche des cycles indépendants dans un graphe}

Le graphe de liaisons est une représentation schématique abstraite de la structure du mécanisme dans lequel les $(p+1)$ pièces sont les sommets et les $\ell$ liaisons sont les arêtes [7]. Dans ce graphe toute liaison $\ell_{\mathrm{i}} \mathrm{du}$ mécanisme est liée par au moins un chemin qui part du bâti. La mise en équations cinématiques du mécanisme est réalisée à partir de l'écriture de la condition de fermeture d'un jeu de $(\ell-p)$ cycles indépendants contenus dans le graphe. Les méthodes systématiques de détermination de ce jeu passe par la détection de l'ensemble de tous les cycles qu'on peut extraire du graphe $[4,8]$. Dans cet ensemble on trouve, entre autres, des cycles non intéressants comme ceux qui comportent plusieurs fois la même arête ou le même sommet ainsi que des cycles qui ne se diffèrent que par leur sens de parcours. Et même dans les méthodes qui ont pu éviter ces genres de cycles, essentiellement l'algorithme de Lagouin [4], le nombre des cycles détectés reste encore important.

Il est clair que la manipulation informatique d'un nombre de cycles largement supérieur au nombre cyclomatique consomme beaucoup de mémoire et nécessite des étapes supplémentaires dans le programme pour se ramener à un jeu de cycles indépendants. Dans un souci de contourner ce problème, Deplanche et Leroy [6] ont développé une méthode systématique de mise en équations cinématiques sans avoir recours à un jeu de 
cycles indépendants. Ce dernier devient, tout simplement, un des résultats de leur méthode. Pour cela ils ont étudié $\ell$ chaînes ouvertes extraites du graphe. Chaque chaîne $C_{i}$ (i variant de 1 à $\ell$ ) possède la particularité que sa liaison terminale est la $i^{\text {ème }}$ liaison du mécanisme. La mise en équation de chaque chaîne $C_{i}$ permet d'obtenir les vitesses absolues de sa pièce terminale en fonction des vitesses relatives des liaisons formant cette chaîne. Chaque chaîne fournit 6 équations, ce qui donne un système de $6 \ell$ équations aux Ic inconnues cinématiques. Parmi les $6 \ell$ seconds membres de ce système on trouve $6 \mathrm{p}$ paramètres qui représentent les vitesses absolues des pièces. Les $6(\ell-p)$ autres seconds membres sont les vitesses absolues des pièces qui se trouvent plusieurs fois terminales car appartenant à des cycles. Ainsi les auteurs retrouvent le nombre cyclomatique $(\ell-p)$ et obtiennent $6(\ell-\mathrm{p})$ équations de fermeture à seconds membres nuls. Chacune d'elles est obtenue uniquement par soustraction des équations initialement écrites dont les seconds membres sont identiques. Cette méthode constitue une solution efficace au problème d'automatisation de la mise en équations cinématiques d'un mécanisme. Cependant, deux points de fond n'ont pas été traités par les auteurs, à savoir la nature des chaînes ouvertes $C_{i}$ et leur mode d'obtention. En effet, nous montrons que pour pouvoir obtenir exactement les $6(\ell-\mathrm{p})$ équations recherchées les $\ell$ chaînes $\mathrm{C}_{\mathrm{i}}$ ne doivent pas être quelconques. De plus, nous pensons que ces chaînes une fois bien définies doivent être déterminées automatiquement et non pas introduites comme des données.

Dans ce paragraphe, nous allons montrer en premier lieu que les chaînes ouvertes que nous avons appelées chemins doivent être les plus courts pour obtenir $(\ell-p)$ cycles et en développant en second lieu une méthode de détermination de ces chemins basée sur la technique de composition latine ou concaténation.

\subsection{Obtention d'un jeu de cycles à partir d'un ensemble de $\ell$ chemins les plus courts}

Soit le chemin $\mathrm{C}_{\mathrm{i}}$, un chemin associé à la liaison $\ell_{\mathrm{i}}$, qui part du bâti et dont la pièce terminale est précédée par la liaison $\ell_{\mathrm{i}}$ (Fig. 1). Ce chemin est désigné par l'ensemble des sommets (pièces) parcourus. Le chemin $\mathrm{C}_{\mathrm{i}}$ de la figure 1 est désigné par $C_{i}=0-1-\ldots-j$, j étant la pièce terminale du chemin $\mathrm{C}_{\mathrm{i}}$. La longueur d'un chemin $\mathrm{C}_{\mathrm{i}}$ représente le nombre des arêtes (liaisons) contenues dans le chemin [9]. Si à toute liaison $\ell_{\mathrm{i}}$ nous associons un seul chemin $\mathrm{C}_{\mathrm{i}}$, nous obtiendrons un ensemble de $\ell$ chemins que nous désignerons par l'ensemble C.

Définition : si deux ou plusieurs chemins ont la même pièce terminale $\mathrm{j}$, ils sont dits complémentaires.

Deux chemins complémentaires forment un seul cycle et $n_{j}$ chemins complémentaires forment $\left(n_{j}-1\right)$ cycles (Fig. 2). Le nombre $n_{j}$ représente aussi le nombre des chemins $\mathrm{C}_{\mathrm{i}}$ où la pièce $\mathrm{j}$ est terminale. Ainsi, le nombre total des cycles détectés systématiquement à partir de

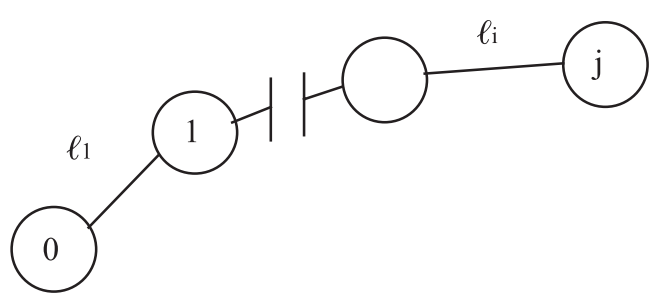

Fig. 1. Chemin $\mathrm{C}_{\mathrm{i}}$ associé à la liaison $\ell_{\mathrm{i}}$.

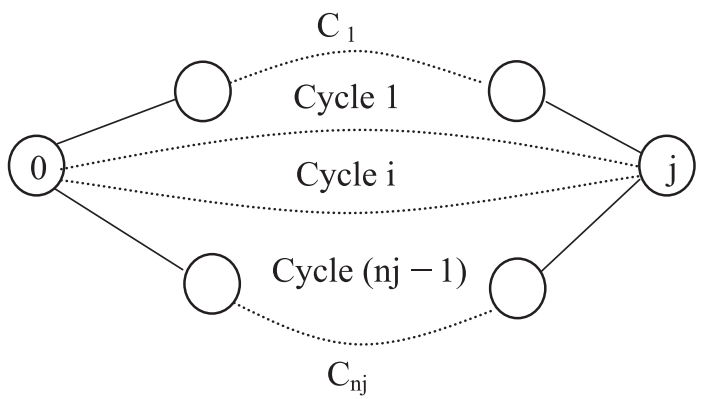

Fig. 2. Obtention des cycles à partir des chemins complémentaires.

l'ensemble $\mathrm{C}$ est la somme des nombres des $\left(\mathrm{n}_{\mathrm{j}}-1\right)$ cycles détectés pour chaque pièce $\mathrm{j}$ :

Nombre total des cycles $=\sum_{j=1}^{p}\left(n_{j}-1\right)=\left(\sum_{j=1}^{p} n_{j}\right)-p$

Puisque nous avons une pièce terminale par chemin $\mathrm{C}_{\mathrm{i}}$, la somme des pièces terminales, indépendamment des $\mathrm{n}_{\mathrm{j}}$, est égal au nombre total des chemins $\mathrm{C}_{\mathrm{i}},\left(\sum_{\mathrm{j}=1}^{\mathrm{p}} \mathrm{n}_{\mathrm{j}}\right)=\ell$. Ce qui donne

$$
\sum_{j=1}^{p}\left(n_{j}-1\right)=\ell-p
$$

Nous retrouvons la relation classique du nombre des cycles indépendants qu'on peut extraire d'un graphe de liaisons. Notre objectif est d'obtenir les $(\ell-p)$ cycles à partir des chemins complémentaires détectés systématiquement dans l'ensemble C. Cet objectif ne peut être réalisé que si $\left(n_{j}-1\right)$ est au minimum égal à zéro. Cela nous amène à exclure obligatoirement le cas où $\mathrm{n}_{\mathrm{j}}=0$, sinon nous aurons dans la somme $\sum_{j=1}^{p}\left(n_{j}-1\right)$ des cycles «négatifs » à comptabiliser. Cette condition signifie que chaque pièce $\mathrm{j}$ doit être terminale au moins une fois dans les chemins de l'ensemble C. Voyons comment cette condition peut être respectée.

Pour les chemins de longueur 1 (comportant une seule arête) la pièce terminale sera une pièce liée au bâti par une liaison directe. Si toutes les liaisons adjacentes au bâti sont associées à des chemins de longueur 1, c'est-à-dire aux chemins les plus courts, les pièces terminales seront les pièces liées au bâti par une liaison directe. Désignons ces pièces par les pièces de niveau 1 . On peut donc dire 


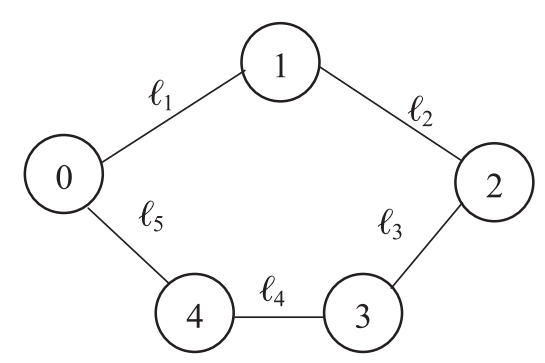

Fig. 3. Graphe à cycle unique.

que toute pièce de niveau 1 est terminale au moins dans un des chemins $\mathrm{C}_{\mathrm{i}}$ de longueur 1.

Essayons de généraliser en désignant les pièces liées au bâti par des chemins les plus courts de longueur k par les pièces de niveau $\mathrm{k}$. Chacune de ces pièces est terminale dans un chemin $\mathrm{C}_{\mathrm{i}}$ de longueur $\mathrm{k}$. Alors, si nous associons à toute liaison $\ell_{\mathrm{i}}$ qui précède une pièce de niveau $\mathrm{k}$ un chemin de longueur $\mathrm{k}$, nous s'assurons que toute pièce du graphe est terminale au moins dans un des chemins $C_{i}$.

Pour illustrer la nécessité de cette condition prenons l'exemple du graphe à cycle unique de la figure 3 . Formons 2 ensembles $\mathrm{C}$ et $\mathrm{C}^{\prime}$ de $\ell$ chemins $\mathrm{C}_{\mathrm{i}}$. L'ensemble $\mathrm{C}$ est constitué des chemins $\mathrm{C}_{\mathrm{i}}$ les plus courts et l'ensemble $\mathrm{C}^{\prime}$ est constitué des chemins $\mathrm{C}_{\mathrm{i}}$ quelconques

$$
\begin{array}{r}
\mathrm{C}=\left\{\mathrm{C}_{1}: 0-1, \mathrm{C}_{2}: 0-1-2, \mathrm{C}_{3}: 0-4-3-2, \mathrm{C}_{4}: 0-4-3,\right. \\
\left.\mathrm{C}_{5}: 0-4\right\} \\
\mathrm{C}^{\prime}=\left\{\mathrm{C}_{1}^{\prime}: 0-1, \mathrm{C}_{2}^{\prime}: 0-1-2, \mathrm{C}_{3}^{\prime}: 0-4-3-2, \mathrm{C}_{4}^{\prime}: 0-1-2-3-4,\right. \\
\left.\mathrm{C}_{5}^{\prime}: 0-4\right\}
\end{array}
$$

L'ensemble $\mathrm{C}$ fournit 2 chemins complémentaires, $\mathrm{C}_{2}$ et $\mathrm{C}_{3}$ qui forment le cycle unique du graphe. Ce qui est vérifié par la relation (1) qui donne $\left(\sum_{\mathrm{j}=1}^{4} \mathrm{n}_{\mathrm{j}}\right)-4=$ $1+2+1+1-4=5-4=1$. Par contre, $\mathrm{C}^{\prime}$ fournit deux couples de chemins complémentaires $\left(\mathrm{C}_{2}^{\prime}, \mathrm{C}_{3}^{\prime}\right)$ et $\left(\mathrm{C}_{4}^{\prime}, \mathrm{C}_{5}^{\prime}\right)$. Ces deux couples vont former deux fois le même cycle.

Dans cet ensemble, la condition $n_{j} \neq 0$ n'est pas respectée pour la pièce 3 qui n'est terminale dans aucun chemin. Ce qui introduit un -1 dans la somme $\sum_{j=1}^{p}\left(n_{j}-1\right)$ et la relation (1), de ce fait, ne vérifie pas le nombre des cycles détectés à partir des chemins complémentaires de l'ensemble $\mathrm{C}^{\prime}$.

Finalement, nous pouvons dire qu'un ensemble de $\ell$ chemins $\mathrm{C}_{\mathrm{i}}$ les plus courts permet d'obtenir exactement $(\ell-p)$ cycles. Ces cycles sont indépendants car chacun d'eux contient au moins un chemin $C_{i}$ qui ne se trouve pas dans les autres cycles.

\subsection{Méthode numérique de formation de l'ensemble C}

Les données nécessaires pour la résolution de ce problème sont les $\mathrm{p}$ pièces, le bâti et les $\ell$ liaisons du mécanisme. Ces données seront représentées dans la matrice latine $[\mathbf{M}][10,11]$. Cette matrice est symétrique,

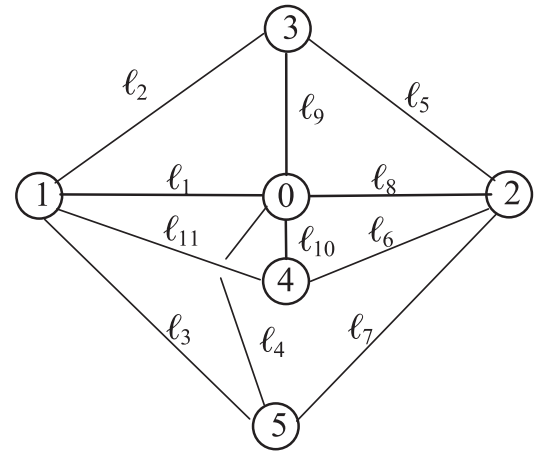

Fig. 4. Graphe multicycles.

d'ordre $(p+1)$. Chaque élément $M[i, j]$ représente le repère de la liaison entre les deux pièces i et $\mathrm{j}$. En cas d'absence de cette liaison, $M[i, j]$ sera égal à l'ensemble vide. Chaque ligne i de $[\mathbf{M}]$ représente les chemins de longueur 1 partant de la pièce $i$.

Dans toute l'opération de détermination de l'ensemble $\mathrm{C}$, les chemins $\mathrm{C}_{\mathrm{i}}$ seront désignés par les arêtes et non pas par les sommets. Cette désignation facilitera la détection des chemins associés aux liaisons $\ell_{i}$. Une fois l'ensemble $\mathrm{C}$ est déterminé, les chemins seront de nouveau désignés par les sommets pour permettre la détection des chemins complémentaires.

Nous allons illustrer la méthode de détermination de l'ensemble C à partir de l'exemple du graphe de la figure 4 . Dans ce graphe le bâti est désigné par le repère (0). La matrice $[\mathbf{M}]$ associée à ce graphe est

$[\mathbf{M}]=$\begin{tabular}{|c|c|c|c|c|c|c|}
\hline & $(1)$ & $(2)$ & $(3)$ & $(4)$ & $(5)$ & $(0)$ \\
\hline$(1)$ & $\emptyset$ & $\emptyset$ & $\ell_{2}$ & $\ell_{11}$ & $\ell_{3}$ & $\ell_{1}$ \\
\hline$(2)$ & $\emptyset$ & $\emptyset$ & $\ell_{5}$ & $\ell_{6}$ & $\ell_{7}$ & $\ell_{8}$ \\
\hline$(3)$ & $\ell_{2}$ & $\ell_{5}$ & $\emptyset$ & $\emptyset$ & $\emptyset$ & $\ell_{9}$ \\
\hline$(4)$ & $\ell_{11}$ & $\ell_{6}$ & $\emptyset$ & $\emptyset$ & $\emptyset$ & $\ell_{10}$ \\
\hline$(5)$ & $\ell_{3}$ & $\ell_{7}$ & $\emptyset$ & $\emptyset$ & $\emptyset$ & $\ell_{4}$ \\
\hline$(0)$ & $\ell_{1}$ & $\ell_{8}$ & $\ell_{9}$ & $\ell_{10}$ & $\ell_{4}$ & $\emptyset$ \\
\hline
\end{tabular}

La dernière ligne de $[\mathbf{M}]$ représente les chemins de longueur 1 partant du bâti. Notons cette ligne

$$
[\mathbf{M}]_{\text {bâti }}^{1}=\begin{array}{|l|l|l|l|l|l|}
\hline \ell_{1} & \ell_{8} & \ell_{9} & \ell_{10} & \ell_{4} & \emptyset \\
\hline
\end{array}
$$

L'exposant 1 correspond à la longueur des chemins, l'indice désigne le sommet de départ. Les chemins de $[\mathbf{M}]_{\text {bâti }}^{1}$ appartiennent tous à l'ensemble $\mathrm{C}$ puisqu'ils sont les chemins les plus courts associés aux liaisons adjacentes au bâti $\ell_{1}, \ell_{4}, \ell_{8}, \ell_{9}$ et $\ell_{10}$. Ces chemins sont $\mathrm{C}_{1}: \ell_{1} ; \mathrm{C}_{4}: \ell_{4}$; $\mathrm{C}_{8}: \ell_{8} ; \mathrm{C}_{9}: \ell_{9} ; \mathrm{C}_{10}: \ell_{10}$.

Il reste pour compléter l'ensemble $\mathrm{C}$, les chemins associés aux liaisons non adjacentes au bâti et qui sont pour notre exemple de longueur 2. Ils sont déterminés à partir de la relation

$$
[\mathbf{M}]_{\text {bâti }}^{2}=[\mathbf{M}]_{\text {bâti }}^{1} \bullet[\mathbf{M}]
$$

où l'opération • est une multiplication particulière dite concaténation mise en œuvre par Kauffmann [10]. On opérera comme dans le calcul matriciel classique, mais au 
lieu d'effectuer des produits, le résultat de toute opération $\mathrm{M}_{\text {bâti }}^{1}[\mathrm{i}] \bullet \mathrm{M}[\mathrm{i}, \mathrm{j}]$ est obtenu de la façon suivante :

- si $\mathrm{M}_{\text {bâti }}^{1}[\mathrm{i}]=\emptyset$ ou $\mathrm{M}[\mathrm{i}, \mathrm{j}]=\emptyset$ alors $\mathrm{M}_{\text {bâti }}^{1}[\mathrm{i}] \bullet \mathrm{M}[\mathrm{i}, \mathrm{j}]=\emptyset$;

- si $\mathrm{M}_{\text {bâti }}^{1}[\mathrm{i}] \cap \mathrm{M}[\mathrm{i}, \mathrm{j}] \neq \emptyset$ alors $\mathrm{M}_{\text {bâti }}^{1}[\mathrm{i}] \bullet \mathrm{M}[\mathrm{i}, \mathrm{j}]=\emptyset$;

- si $\mathrm{M}_{\text {bâti }}^{1}[\mathrm{i}] \cap \mathrm{M}[\mathrm{i}, \mathrm{j}]=\emptyset$ et $\mathrm{M}_{\text {bâti }}^{1}[\mathrm{i}] \neq \emptyset, \mathrm{M}[\mathrm{i}, \mathrm{j}] \neq \emptyset$ alors $\mathrm{M}_{\text {bâti }}^{1}[\mathrm{i}] \bullet \mathrm{M}[\mathrm{i}, \mathrm{j}]$ donne le chemin $\mathrm{M}_{\text {bâti }}^{2}[\mathrm{i}, \mathrm{k}]$ composé $\mathrm{du}$ chemin de $\mathrm{M}_{\text {bâti }}^{1}[\mathrm{i}]$ plus le chemin de $\mathrm{M}[\mathrm{i}, \mathrm{j}]$.

Ainsi la multiplication de $[\mathbf{M}]_{\text {bâti }}^{1}$ par la première colonne de $[\mathbf{M}]$ donne

$$
\begin{aligned}
& \ell_{1} \bullet \emptyset=\emptyset \\
& \ell_{8} \bullet \emptyset=\emptyset \\
& \ell_{9} \bullet \ell_{2}=\ell_{9}-\ell_{2} \\
& \ell_{10} \bullet \ell_{11}=\ell_{10}-\ell_{11} \\
& \ell_{4} \bullet \ell_{3}=\ell_{4}-\ell_{3} \\
& \emptyset \bullet \ell_{1}=\emptyset
\end{aligned}
$$

On obtient les trois chemins $\ell_{9}-\ell_{2}, \ell_{10}-\ell_{11}$ et $\ell_{4}-\ell_{3}$ qui partent du bâti et qui arrivent tous à la pièce (1). La multiplication de $[\mathbf{M}]_{\text {batti }}^{1}$ par toutes les colonnes de $[\mathbf{M}]$ donne les chemins suivants :

$$
[\mathbf{M}]_{\text {bâti }}^{2}=\begin{array}{c|c|c|c|c|c|}
\hline \ell_{9}-\ell_{2} & \ell_{9}-\ell_{5} & \ell_{1}-\ell_{2} & \ell_{1}-\ell_{11} & \ell_{1}-\ell_{3} & \emptyset \\
\ell_{10}-\ell_{11} & \ell_{10}-\ell_{6} & \ell_{8}-\ell_{5} & \ell_{8}-\ell_{6} & \ell_{8}-\ell_{7} & \\
\ell_{4}-\ell_{3} & \ell_{4}-\ell_{7} & & & & \\
\hline
\end{array}
$$

On remarque que pour une même liaison $\ell_{\mathrm{i}},[\mathbf{M}]_{\text {bâti }}^{2}$ fournit plus d'un chemin $\mathrm{C}_{\mathrm{i}}$. Parmi ces chemins, on doit prendre un quelconque pour l'ensemble C. Le plus simple est de prendre le premier chemin trouvé.

Les chemins considérés à partir de $[\mathbf{M}]_{\text {bâti }}^{2}$ sont $\mathrm{C}_{2}$ : $\ell_{9}-\ell_{2}, \mathrm{C}_{3}: \ell_{4}-\ell_{3}, \mathrm{C}_{5}: \ell_{8}-\ell_{5}, \mathrm{C}_{6}: \ell_{10}-\ell_{6}, \mathrm{C}_{7}: \ell_{8}-\ell_{7}$, et $\mathrm{C}_{11}: \ell_{10}-\ell_{11}$. L'ensemble $\mathrm{C}$ obtenu est

$$
\begin{array}{r}
\mathrm{C}=\left\{\mathrm{C}_{1}: \ell_{1}, \mathrm{C}_{2}: \ell_{9}-\ell_{2}, \mathrm{C}_{3}: \ell_{4}-\ell_{3}, \mathrm{C}_{4}: \ell_{4}, \mathrm{C}_{5}: \ell_{8}-\ell_{5},\right. \\
\mathrm{C}_{6}: \ell_{10} \ell_{6}, \mathrm{C}_{7}: \ell_{8}-\ell_{7}, \mathrm{C}_{8}: \ell_{8}, \mathrm{C}_{9}: \ell_{9}, \mathrm{C}_{10}: \ell_{10}, \\
\left.\mathrm{C}_{11}: \ell_{10} \ell_{11}\right\}
\end{array}
$$

En exprimant les chemins $C_{i}$ en fonction des repères des pièces l'ensemble $\mathrm{C}$ devient

$$
\begin{array}{r}
\mathrm{C}=\left\{\mathrm{C}_{1}: 0-1, \mathrm{C}_{2}: 0-3-1, \mathrm{C}_{3}: 0-5-1, \mathrm{C}_{4}: 0-5, \mathrm{C}_{5}: 0-2-3,\right. \\
\mathrm{C}_{6}: 0-4-2, \mathrm{C}_{7}: 0-2-5, \mathrm{C}_{8}: 0-2, \mathrm{C}_{9}: 0-3, \mathrm{C}_{10}: 0-4, \\
\left.\mathrm{C}_{11}: 0-4-1\right\}
\end{array}
$$

D’une façon générale la méthode de concaténation de Kauffman permet d'obtenir les chemins de longueur $x$ à partir des chemins de longueur $x-1$ selon la relation suivante

$$
[\mathbf{M}]_{\text {bâti }}^{x}=[\mathbf{M}]_{\text {bâti }}^{x-1} \bullet[\mathbf{M}]
$$

L'opération de concaténation se déroule de la même façon que pour celle exposée pour $x=2$ dans l'exemple précédent. Cependant $[\mathbf{M}]_{\text {bâti }}^{x-1}[\mathrm{i}]$ peut contenir plus d'un chemin, dans ce cas tout chemin $[\mathbf{M}]_{\text {bâti }}^{x-1}[\mathrm{i}, \mathrm{a}]$ doit être multiplié par $\mathrm{M}[\mathrm{i}, \mathrm{j}]$.

Le processus de concaténation est arrêté dès que l'ensemble C est complètement déterminé.
Tableau 1. Les 6 cycles indépendants du graphe de la figure 4 .

\begin{tabular}{cl}
\hline Couple de chemins complémentaires & Cycle obtenu \\
\hline$\left(\mathrm{C}_{1}, \mathrm{C}_{2}\right)$ & Cycle $1: 0-1-3-0$ \\
$\left(\mathrm{C}_{1}, \mathrm{C}_{3}\right)$ & Cycle $2: 0-1-5-0$ \\
$\left(\mathrm{C}_{1}, \mathrm{C}_{11}\right)$ & Cycle $3: 0-4-1-0$ \\
$\left(\mathrm{C}_{4}, \mathrm{C}_{7}\right)$ & Cycle $4: 0-5-2-0$ \\
$\left(\mathrm{C}_{5}, \mathrm{C}_{9}\right)$ & Cycle $5: 0-2-3-0$ \\
$\left(\mathrm{C}_{6}, \mathrm{C}_{8}\right)$ & Cycle $6: 0-4-2-0$ \\
\hline
\end{tabular}

\subsection{Formation d'un jeu de cycles indépendants}

À partir de l'ensemble C, nous cherchons les sous-ensembles des chemins complémentaires. Un sousensemble de $n_{j}$ chemins fournit $\left(n_{j}-1\right)$ cycles. Pour notre exemple d'illustration, l'ensemble $\mathrm{C}$ nous fournit les sousensembles des chemins complémentaires suivants :

- 4 chemins $\mathrm{C}_{1}, \mathrm{C}_{2}, \mathrm{C}_{3}, \mathrm{C}_{11}$ de pièce terminale 1 qui forment 3 cycles ;

- 2 chemins $\mathrm{C}_{4}, \mathrm{C}_{7}$ de pièce terminale 5 qui forment 1 cycle;

- 2 chemins $\mathrm{C}_{5}, \mathrm{C}_{9}$ de pièce terminale 3 qui forment 1 cycle;

- 2 chemins $\mathrm{C}_{6}, \mathrm{C}_{8}$ de pièce terminale 2 qui forment 1 cycle.

Nous obtenons au total les 6 cycles du tableau 1 .

\section{Mise en équation systématique}

La mise en équation de chacun des deux chemins complémentaires $\mathrm{C}_{\mathrm{i}}$ et $\mathrm{C}_{\mathrm{j}}$ de la figure 5, donne le mouvement de la pièce terminale t par rapport au bâti 0 . Le torseur cinématique $\left\{\vartheta_{\mathrm{t} / 0}\right\}$ de ce mouvement s'écrit en considérant le chemin $\mathrm{C}_{\mathrm{i}}$ :

$$
\left\{\vartheta_{\mathrm{t} / 0}\right\}_{\mathrm{o}}=\left\{\vartheta_{\mathrm{t} / x}\right\}_{\mathrm{o}}+\left\{\vartheta_{x / x-1}\right\}_{\mathrm{o}}+\ldots+\left\{\vartheta_{1 / 0}\right\}_{\mathrm{o}}
$$

ou en considérant le chemin $\mathrm{C}_{\mathrm{j}}$

$\left\{\vartheta_{\mathrm{t} / 0}\right\}_{\mathrm{o}}=\left\{\vartheta_{\mathrm{t} / x+\mathrm{n}}\right\}_{\mathrm{o}}+\left\{\vartheta_{x+\mathrm{n} / x+\mathrm{n}-1}\right\}_{\mathrm{o}}+\ldots+\left\{\vartheta_{x+1 / 0}\right\}_{\mathrm{o}}$

La soustraction des deux relations (3) et (4) nous donne l'équation (5) de fermeture du cycle

$$
\begin{aligned}
\left\{\vartheta_{\mathrm{t} / x}\right\}_{\mathrm{o}} & +\left\{\vartheta_{x / x-1}\right\}_{\mathrm{o}}+\ldots+\left\{\vartheta_{1 / 0}\right\}_{\mathrm{o}}-\left\{\vartheta_{\mathrm{t} / x+n}\right\}_{\mathrm{o}} \\
& -\left\{\vartheta_{x+\mathrm{n} / x+\mathrm{n}-1}\right\}_{\mathrm{o}}-\ldots-\left\{\vartheta_{x+1 / 0}\right\}_{\mathrm{o}}=\{0\}
\end{aligned}
$$

Pour l'ensemble des $(\ell-p)$ cycles indépendants du mécanisme, on aura un système de $6 \times(\ell-\mathrm{p})$ équations à $\sum_{\mathrm{i}=1}^{\ell} \mathrm{d} \ell_{\mathrm{i}}$ inconnues cinématiques, avec $\mathrm{d} \ell_{\mathrm{i}}$ le degré de liberté de la liaison $\ell_{\mathrm{i}}$. 


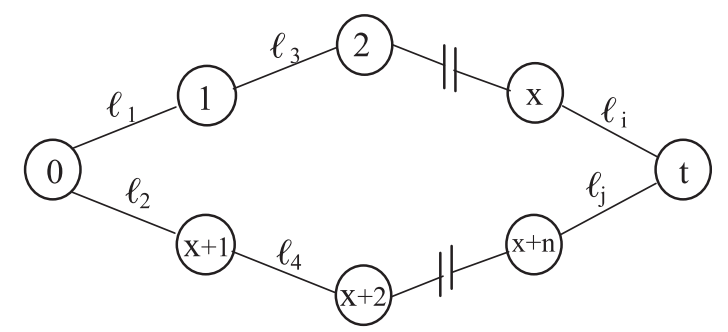

Fig. 5. Cycle unique.

Le torseur cinématique associé à la liaison entre les pièces $\mathrm{i}$ et $\mathrm{j}$, est défini dans un repère local $\mathrm{R}_{\text {local }}\left(\mathrm{O}^{\prime}, \mathbf{u}\right.$, $\mathbf{v}, \mathbf{w})$ par

$$
\left\{\vartheta_{\mathrm{j} / \mathrm{i}}\right\}=\left\{\begin{array}{c}
\alpha_{\mathrm{ji}} \\
\beta_{\mathrm{ji}} \\
\gamma_{\mathrm{ji}} \\
\mathbf{u}_{\mathrm{ji}} \\
\mathbf{v}_{\mathrm{ji}} \\
\mathbf{w}_{\mathrm{ji}}
\end{array}\right\}
$$

Dans l'équation (5), les torseurs des liaisons sont écrits dans un repère global à définir. Le torseur cinématique de chaque liaison est dans ces conditions représenté par la matrice rectangulaire $\left[\mathbf{T}_{\mathrm{c}}(\mathbf{j} / \mathbf{i})\right]$ de dimensions variables de $(6 \times 1)$ jusqu'à $(6 \times 5)$ selon le nombre des inconnues cinématiques du torseur $\left\{\vartheta_{\mathrm{j} / \mathrm{i}}\right\}$. D'une façon générale la matrice $\left[\mathbf{T}_{\mathrm{c}}(\mathbf{j} / \mathbf{i})\right]$ s'écrit $[11]$ :

$$
\begin{aligned}
{\left[\mathbf{T}_{\mathrm{c}}(\mathbf{j} / \mathbf{i})\right] } & =\left[\mathbf{M}_{\mathrm{t}}\right]\left[\mathbf{M}_{\mathrm{p}}\right] \cdot[\mathbf{I}]\left\{\vartheta_{\mathrm{j} / \mathrm{i}}\right\} \\
& =\left[\begin{array}{cc}
\mathbf{1} & 0 \\
\mathbf{m}_{\mathrm{t}} & \mathbf{1}
\end{array}\right]\left[\begin{array}{cc}
\mathbf{m}_{\mathrm{p}} & 0 \\
0 & \mathbf{m}_{\mathrm{p}}
\end{array}\right][\mathbf{I}]\left\{\vartheta_{\mathrm{j} / \mathrm{i}}\right\}
\end{aligned}
$$

où

$\left[\mathbf{m}_{\mathrm{t}}\right]$ : matrice $(3 \times 3)$ de transport du torseur à l'origine du repère global,

$\left[\mathbf{m}_{\mathrm{p}}\right]$ : matrice $(3 \times 3)$ de passage du repère local au repère global,

$[\mathbf{I}]$ : matrice identité $(6 \times 6)$.

L'annexe illustre la forme des matrices $\left[\mathbf{m}_{\mathrm{t}}\right]$ et $\left[\mathbf{m}_{\mathrm{p}}\right]$ ainsi que la forme générale de $\left[\mathbf{T}_{\mathrm{c}}(\mathbf{j} / \mathbf{i})\right]$.

Le remplacement des $\left\{\vartheta_{\mathrm{j} / \mathrm{i}}\right\}$ par $\left[\mathbf{T}_{\mathrm{c}}(\mathbf{j} / \mathbf{i})\right]$ dans l'équation (5) nous donne

$$
\begin{array}{r}
{\left[\mathbf{T}_{\mathrm{c}}(\mathbf{t} / \mathbf{x})\right]+\left[\mathbf{T}_{\mathrm{c}}(\mathbf{x} / \mathbf{x}-\mathbf{1})\right]+\ldots+\left[\mathbf{T}_{\mathrm{c}}(\mathbf{1} / \mathbf{0})\right]-\left[\mathbf{T}_{\mathrm{c}}(\mathbf{t} / \mathbf{x}+\mathbf{n})\right]} \\
-\left[\mathbf{T}_{\mathrm{c}}(\mathbf{x}+\mathbf{n} / \mathbf{x}+\mathbf{n}-\mathbf{1})\right]-\ldots-\left[\mathbf{T}_{\mathrm{c}}(\mathbf{x}+\mathbf{1} / \mathbf{0})\right]=\{\mathbf{0}\}
\end{array}
$$

La mise en équations cinématiques est basée sur une technique d'assemblage $[12,14]$ des matrices $\left[\mathbf{T}_{\mathrm{c}}(\mathbf{j} / \mathbf{i})\right]$ associés aux liaisons formant le cycle. La matrice du système cinématique est ainsi obtenue automatiquement et progressivement par bloc de 6 lignes en suivant l'ordre de détection des cycles indépendants.

\section{Analyse du système cinématique}

L'écriture des $(\ell-\mathrm{p})$ relations (8) relatives à la fermeture des cycles indépendants permet d'obtenir le système linéaire homogène suivant :

$$
[\mathbf{E}] \cdot\{\mathbf{V}\}=\{\mathbf{0}\}
$$

où

$\{\mathbf{V}\}$ : vecteur des inconnues cinématiques de dimension $\left(\mathrm{Ic}=\sum_{\mathrm{i}=1}^{\ell} \mathrm{d} \ell_{\mathrm{i}}\right)$

$[\mathbf{E}]$ : matrice rectangulaire de dimensions $(6(\ell-\mathrm{p}) \times$ Ic).

La triangularisation du système par la méthode de pivot total de Gauss nous donne

$$
\left[\mathbf{E}_{1}\right]\left\{\mathbf{V}_{1}\right\}+\left[\mathbf{E}_{2}\right]\{\mathbf{W}\}=\{\mathbf{0}\}
$$

avec

$\left[\mathbf{E}_{1}\right]$ matrice carrée triangulaire supérieure d'ordre r ; $\mathrm{r}$ : rang de $[\mathbf{E}]$,

$\left[\mathbf{E}_{2}\right]$ matrice rectangulaire de dimension $(\mathrm{r} \times \mathrm{m}) ; \mathrm{m}$ : nombre d'inconnues non principales ou degré de mobilité du mécanisme,

$\left\{\mathbf{V}_{1}\right\}$ vecteur de dimension $\mathrm{r}$ des inconnues cinématiques principales,

$\{\mathbf{W}\}$ vecteur de dimension $\mathrm{m}$ des paramètres cinématiques indépendants.

La relation entre $\left\{\mathbf{V}_{1}\right\}$ et $\{\mathbf{W}\}$ s'écrit

$$
\left\{\mathbf{V}_{1}\right\}=-\left[\mathbf{E}_{1}\right]^{-1}\left[\mathbf{E}_{2}\right]\{\mathbf{W}\}=-\left[\mathbf{E}_{3}\right]\{\mathbf{W}\}
$$

Le système (11) ne peut être déterminé que si les m paramètres cinématiques indépendants formant le vecteur $\{\mathbf{W}\}$ sont donnés. Cependant, on peut distinguer parmi les inconnues de $\left\{\mathbf{V}_{1}\right\}$, ceux qui ne dépendent pas de $\{\mathbf{W}\}$ et qui sont donc nulles. Le système (11) peut être écrit dans ce cas

$$
\left\{\mathbf{V}_{1}\right\}=\left\{\begin{array}{c}
\mathbf{0} \\
\mathbf{V}_{1}^{\prime}
\end{array}\right\}=-\left[\begin{array}{c}
\mathbf{0} \\
\mathbf{E}_{3}^{\prime}
\end{array}\right]\{\mathbf{W}\}
$$

$\left[\mathbf{E}_{3}^{\prime}\right]$ : partie non nulle de $\left[\mathbf{E}_{3}\right]$.

Le système qui reste à résoudre est

$$
\left\{\mathbf{V}_{1}^{\prime}\right\}=-\left[\mathbf{E}_{3}^{\prime}\right]\{\mathbf{W}\}
$$

Notons $\mathrm{r}^{\prime \prime}$ la dimension de $\left\{\mathbf{V}_{1}^{\prime}\right\}$.

\subsection{Exemple}

Soit le mécanisme d'essuie-glace représenté par la figure 6. Les 9 liaisons du mécanisme sont décrites dans le tableau 2. La liaison $\ell_{5}$ entre le pignon 4 et la crémaillère 3 a été considérée dans une première approximation comme un appui ponctuel. Vu que nous adoptons l'hypothèse des liaisons parfaites, le frottement est négligé; de ce fait le système roue et vis sans fin est réversible. L'analyse cinématique du mécanisme selon le 
Tableau 2. Liaisons du mécanisme d'essuie glaces.

\begin{tabular}{|c|c|c|c|c|c|}
\hline Liaison & Type & Pièces & Position & $\begin{array}{l}\text { repère local } \\
\left(\mathrm{R}_{\text {local }}\right)\end{array}$ & Torseur cinématique/ $\mathrm{R}_{\text {local }}$ \\
\hline$\ell_{1}$ & pivot & $0-1$ & $\left(\begin{array}{l}0 \\
0 \\
0\end{array}\right)$ & {$\left[\begin{array}{lll}1 & 0 & 0 \\
0 & 1 & 0 \\
0 & 0 & 1\end{array}\right]$} & $\left\{\vartheta_{1 / 0}\right\}^{\mathrm{T}}=\left\{\alpha_{10}, 0,0,0,0,0\right\}$ \\
\hline$\ell_{2}$ & Appui ponctuel & $2-1$ & $\left(\begin{array}{c}24 \\
0 \\
4\end{array}\right)$ & $\begin{array}{ccc}-0.34 & 0 & -0.94 \\
0 & 1 & 0 \\
0.94 & 0 & -0.34\end{array}$ & $\left\{\vartheta_{2 / 1}\right\}^{\mathrm{T}}=\left\{\alpha_{21}, \beta_{21}, \gamma_{21}, \mathrm{u}_{21}, \mathrm{v}_{21}, 0\right\}$ \\
\hline$\ell_{3}$ & pivot & $0-2$ & $\left(\begin{array}{c}24 \\
0 \\
-22.5\end{array}\right)$ & {$\left[\begin{array}{ccc}0 & -1 & 0 \\
1 & 0 & 0 \\
0 & 0 & 1\end{array}\right]$} & $\left\{\vartheta_{2 / 0}\right\}^{\mathrm{T}}=\left\{\alpha_{20}, 0,0,0,0,0\right\}$ \\
\hline$\ell_{4}$ & pivot & $2-3$ & $\left(\begin{array}{c}21.5 \\
9 \\
-28.5\end{array}\right)$ & $\begin{array}{ccc}0 & -1 & 0 \\
1 & 0 & 0 \\
0 & 0 & 1 \\
\end{array}$ & $\left\{\vartheta_{3 / 2}\right\}^{\mathrm{T}}=\left\{\alpha_{32}, 0,0,0,0,0\right\}$ \\
\hline$\ell_{5}$ & Appui ponctuel & $4-3$ & $\left(\begin{array}{c}41.8 \\
9 \\
-51.2\end{array}\right)$ & $\left.\begin{array}{ccc}0 & 0 & 1 \\
0 & 1 & 0 \\
-1 & 0 & 0\end{array}\right]$ & $\left\{\vartheta_{3 / 4}\right\}^{\mathrm{T}}=\left\{\alpha_{34}, \beta_{34}, \gamma_{34}, \mathrm{u}_{34}, \mathrm{v}_{34}, 0\right\}$ \\
\hline$\ell_{6}$ & pivot & $0-4$ & $\left.\begin{array}{c}43.25 \\
9 \\
-47.25\end{array}\right)$ & $\left.\begin{array}{ccc}0 & -1 & 0 \\
1 & 0 & 0 \\
0 & 0 & 1\end{array}\right]$ & $\left\{\vartheta_{4 / 0}\right\}^{\mathrm{T}}=\left\{\alpha_{40}, 0,0,0,0,0\right\}$ \\
\hline$\ell_{7}$ & pivot & $4-5$ & $\left(\begin{array}{c}43.2 \\
17 \\
-47.2\end{array}\right)$ & {$\left[\begin{array}{ccc}0 & -1 & 0 \\
1 & 0 & 0 \\
0 & 0 & 1\end{array}\right]$} & $\left\{\vartheta_{5 / 4}\right\}^{\mathrm{T}}=\left\{\alpha_{54}, 0,0,0,0,0\right\}$ \\
\hline$\ell_{8}$ & pivot & $5-6$ & $\left(\begin{array}{c}48.2 \\
9 \\
-34\end{array}\right)$ & {$\left[\begin{array}{ccc}0 & -1 & 0 \\
1 & 0 & 0 \\
0 & 0 & 1\end{array}\right]$} & $\left\{\vartheta_{6 / 5}\right\}^{\mathrm{T}}=\left\{\alpha_{65}, 0,0,0,0,0\right\}$ \\
\hline$\ell_{9}$ & Appui ponctuel & $3-6$ & $\left(\begin{array}{c}40 \\
9 \\
-37.7\end{array}\right)$ & $\begin{array}{ccc}-0.94 & 0 & 0.34 \\
0 & -1 & 0 \\
0.34 & 0 & 0.94\end{array}$ & $\left\{\vartheta_{6 / 3}\right\}^{\mathrm{T}}=\left\{\alpha_{63}, \beta_{63}, \gamma_{63}, \mathrm{u}_{63}, \mathrm{v}_{63}, 0\right\}$ \\
\hline
\end{tabular}

processus décrit précédemment nous donne les résultats suivants :

\section{Caractéristiques générales}

Nombre de pièces : 6

Degré de mobilité : 3

Degré d'hyprestaticité : 0

Vitesses nulles

- Liaison $\ell_{5}: \alpha_{34}-\gamma_{34}-\mathbf{v}_{34}$

- Liaison $\ell_{9}: \alpha_{63}-\gamma_{63}-\mathbf{v}_{63}$

\subsection{Identification des paramètres cinématiques indépendants}

Le vecteur $\{\mathbf{W}\}$ du système (13) comprend les paramètres cinématiques indépendants dégagés par l'algorithme de triangularisation. Cependant, il est important, dans une phase d'analyse d'un mécanisme,
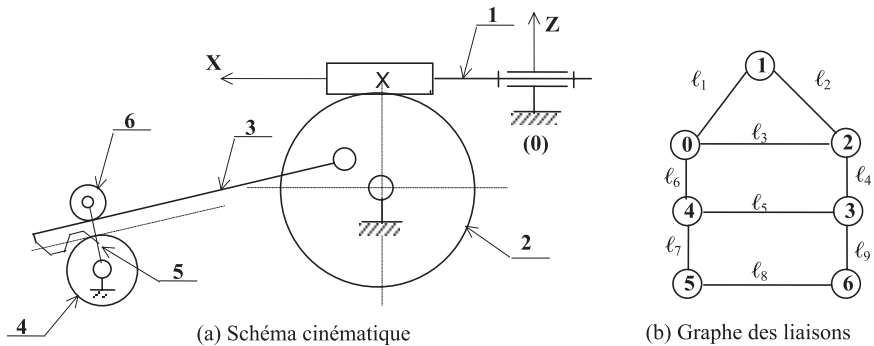

Fig. 6. Mécanisme d'essuie-glace.

d'explorer toutes les formations possibles du vecteur $\{\mathbf{W}\}$ afin de déterminer toutes les possibilités cinématiques du mécanisme. Nous appelons jeu de paramètres cinématiques une des formations possibles du vecteur $\{\mathbf{W}\}$. Dans ce paragraphe, Nous allons présenter, une méthode numérique qui permet la détermination de l'ensemble de ces jeux et ce relativement à une configuration donnée du mécanisme. 


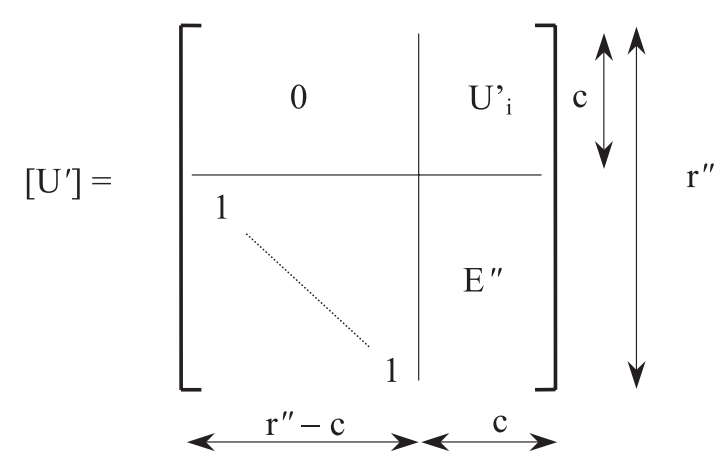

Fig. 7. Constitution de la matrice $\left[\mathrm{U}^{\prime}\right]$ avec c variant de 1 à $\mathrm{m}$.

\subsubsection{Détermination de l'ensemble des jeux de paramètres cinématiques indépendants}

L'ensemble des jeux des paramètres cinématiques indépendants, est composé de jeux valides parmi tous les jeux arbitraires de $m$ paramètres qu'on peut former à partir des $\left(\mathrm{r}^{\prime \prime}+\mathrm{m}\right)$ inconnues des vecteurs $\left\{\mathbf{V}_{1}^{\prime}\right\}$ et $\{\mathbf{W}\}$.

La relation (13) s'écrit aussi

$$
[\mathbf{1}]\left\{\mathbf{V}_{1}^{\prime}\right\}=-\left[\mathbf{E}_{3}^{\prime}\right]\{\mathbf{W}\}
$$

À partir de cette relation, un jeu arbitraire $\left\{\mathbf{W}_{\mathrm{i}}\right\}$ est une permutation de $\mathrm{c}$ inconnues $(1 \leq \mathrm{c} \leq \mathrm{m})$ entre les composants $\left\{\mathbf{V}_{1}^{\prime}\right\}$ et ses colonnes correspondants dans [1] et les composants de $\{\mathbf{W}\}$ et ses colonnes correspondant dans $\left[\mathbf{E}_{3}^{\prime}\right]$. La relation (13) devient

$$
\left[\mathbf{U}_{\mathrm{i}}\right]\left\{\mathbf{V}_{1 \mathrm{i}}^{\prime}\right\}=-\left[\mathbf{E}_{3 \mathrm{i}}^{\prime}\right]\left\{\mathbf{W}_{\mathrm{i}}\right\}
$$

Pour que le jeu $\left\{\mathbf{W}_{\mathrm{i}}\right\}$ soit valide, il faut que la matrice $\left[\mathbf{U}_{\mathrm{i}}\right]$ soit de rang complet. La matrice $\left[\mathbf{U}_{\mathrm{i}}\right]$ est formée de $\left(\mathrm{r}^{\prime \prime}-\mathrm{c}\right)$ colonnes de la matrice identité et de c colonnes de la matrice $\left[\mathbf{E}_{3}^{\prime}\right]$, comme c'est illustré par la figure 7 . Le calcul du déterminant de $\left[\mathbf{U}_{\mathrm{i}}\right]$ sera réduit à celui du déterminant de la sous-matrice carrée $\left[\mathbf{U}_{\mathbf{i}}^{\prime}\right]$ extraite de $\left[\mathbf{E}_{3}^{\prime}\right]$ et de dimension $\mathrm{c}$ variant de 1 à $\mathrm{m}$.

$$
\operatorname{dét}\left[\mathbf{U}^{\prime}\right]= \pm \operatorname{dét}\left[\mathbf{U}_{\mathrm{i}}^{\prime}\right]
$$

Voyons l'application de ce calcul sur l'exemple de l'essuie-glace. Le système (13) obtenu est :

$[\mathbf{1}]\left\{\begin{array}{l}\beta_{34} \\ \alpha_{65} \\ \alpha_{20} \\ \alpha_{21} \\ \beta_{21} \\ \alpha_{40} \\ \alpha_{32} \\ \gamma_{21} \\ \alpha_{54} \\ \alpha_{10} \\ \beta_{63} \\ \mathbf{u}_{21}\end{array}\right\}=-\left[\begin{array}{ccc}-0.012 & 0 & 0.244 \\ -0.00005 & -0.069 & 0.115 \\ 0.005 & 0 & 0 \\ 0.085 & 0 & 0 \\ -0.025 & 0 & 0 \\ 0.137 & 0 & -0.316 \\ -0.003 & 0 & -0.071 \\ 0.232 & 0 & 0 \\ -0.019 & 0.071 & 0.431 \\ -0.249 & 0 & 0 \\ 0.0075 & -0.002 & -0.302 \\ 0.032 & 0 & 0\end{array}\right]\left\{\begin{array}{l}\mathbf{v}_{21} \\ \mathbf{u}_{63} \\ \mathbf{u}_{34}\end{array}\right\}$

Soit le jeu arbitraire à tester $\left\{\alpha_{20} \beta_{34} \alpha_{65}\right\}$. Les 3 inconnues $\mathrm{v}_{21}, \mathrm{u}_{34}$ et $\mathrm{u}_{63}$ vont passer dans $\left\{\mathbf{V}_{1}^{\prime}\right\}$. Nous obtenons le système suivant :

$$
\left[\begin{array}{cccccccccccccc}
-0.012 & 0 & 0.244 & 0 & 0 & 0 & 0 & 0 & 0 & 0 & 0 & 0 \\
-0.0005 & -0.069 & 0.115 & 0 & 0 & 0 & 0 & 0 & 0 & 0 & 0 & 0 \\
0.005 & 0 & 0 & 0 & 0 & 0 & 0 & 0 & 0 & 0 & 0 & 0 \\
0.085 & 0 & 0 & 1 & 0 & 0 & 0 & 0 & 0 & 0 & 0 & 0 \\
-0.025 & 0 & 0 & 0 & 1 & 0 & 0 & 0 & 0 & 0 & 0 & 0 \\
0.137 & 0 & -0.316 & 0 & 0 & 1 & 0 & 0 & 0 & 0 & 0 & 0 \\
-0.003 & 0 & -0.071 & 0 & 0 & 0 & 1 & 0 & 0 & 0 & 0 & 0 \\
0.232 & 0 & 0 & 0 & 0 & 0 & 0 & 1 & 0 & 0 & 0 & 0 \\
-0.019 & 0.071 & 0.431 & 0 & 0 & 0 & 0 & 0 & 1 & 0 & 0 & 0 \\
-0.249 & 0 & 0 & 0 & 0 & 0 & 0 & 0 & 0 & 1 & 0 & 0 \\
0.0075 & -0.002 & -0.302 & 0 & 0 & 0 & 0 & 0 & 0 & 0 & 1 & 0 \\
0.032 & 0 & 0 & 0 & 0 & 0 & 0 & 0 & 0 & 0 & 0 & 1
\end{array}\right]\left\{\begin{array}{l}
\mathbf{v}_{21} \\
\mathbf{u}_{63} \\
\mathbf{u}_{34} \\
\alpha_{21} \\
\beta_{21} \\
\alpha_{40} \\
\alpha_{32} \\
\gamma_{21} \\
\alpha_{54} \\
\alpha_{10} \\
\beta_{63} \\
\mathbf{u}_{21}
\end{array}\right\}=
$$

$$
-\left[\begin{array}{lll}
1 & 0 & 0 \\
0 & 1 & 0 \\
0 & 0 & 1 \\
0 & 0 & 0 \\
0 & 0 & 0 \\
0 & 0 & 0 \\
0 & 0 & 0 \\
0 & 0 & 0 \\
0 & 0 & 0 \\
0 & 0 & 0 \\
0 & 0 & 0 \\
0 & 0 & 0
\end{array}\right]\left\{\begin{array}{l}
\beta_{34} \\
\alpha_{65} \\
\alpha_{20}
\end{array}\right\}
$$

Pour que le jeu $\left\{\alpha_{20} \beta_{34} \alpha_{65}\right\}$ soit valide, il faut que le déterminant de la matrice $\left[\mathrm{U}^{\prime}\right]$ soit non nul. Ce déterminant est égal au déterminant de la matrice $\left[\mathrm{U}_{\mathrm{i}}^{\prime}\right]$ composée des intersections des 3 premières lignes avec les trois premières colonnes.

$$
\begin{aligned}
\operatorname{dét}\left[\mathrm{U}^{\prime}\right] & =\operatorname{dét}\left[U_{i}^{\prime}\right]=+\operatorname{dét}\left[\begin{array}{ccc}
-0.012 & 0 & 0.244 \\
-0.0005 & -0.069 & 0.115 \\
0.005 & 0 & 0
\end{array}\right] \\
& =0.0193
\end{aligned}
$$

Ce déterminant est non nul et le jeu $\left\{\alpha_{20} \beta_{34} \alpha_{65}\right\}$ est donc valable.

En testant de cette façon tous les jeux arbitraires $\left\{\mathbf{W}_{\mathrm{i}}\right\}$, nous obtenons l'ensemble de tous les jeux valables des paramètres cinématiques indépendants. L'ensemble complet des jeux du mécanisme de l'essuie glace est donné par le tableau 3.

\subsubsection{Méthode pratique du choix d'un jeu de paramètres cinématiques indépendants}

Nous remarquons que le nombre élevé des jeux du tableau 3 rend difficile une bonne exploitation du résultat obtenu. Cette difficulté va être d'autant plus grande que le degré de mobilité est plus important. Dans un but de saisir facilement le jeu convenable de paramètres 
Tableau 3. Liste des jeux des paramètres cinématiques indépendants.

\begin{tabular}{|c|c|c|c|c|}
\hline$\left\{\alpha_{10} \alpha_{32} \alpha_{54}\right\}$ & $\left\{\alpha_{21} \alpha_{32} \alpha_{54}\right\}$ & $\left\{\gamma_{21} \alpha_{54} \alpha_{65}\right\}$ & $\left\{v_{21} \alpha_{40} \beta_{63}\right\}$ & $\left\{\alpha_{65} \alpha_{32} \mathbf{u}_{34}\right\}$ \\
\hline$\left\{\alpha_{21} \mathbf{u}_{34} \alpha_{54}\right\}$ & $\left\{\alpha_{21} \alpha_{32} \alpha_{65}\right\}$ & $\left\{\gamma_{21} \alpha_{54} \beta_{63}\right\}$ & $\left\{\begin{array}{lll}\mathbf{v}_{21} & \alpha_{40} & \mathbf{u}_{63}\end{array}\right\}$ & $\left\{\alpha_{65} \alpha_{32} \alpha_{40}\right\}$ \\
\hline$\left\{\alpha_{21} u_{34} \alpha_{65}\right\}$ & $\left\{\alpha_{21} \alpha_{32} \beta_{63}\right\}$ & $\left\{\gamma_{21} \alpha_{54} \mathbf{u}_{63}\right\}$ & $\left\{\alpha_{10} \alpha_{54} \alpha_{65}\right\}$ & $\left\{\alpha_{65} \beta_{34} \mathbf{u}_{34}\right\}$ \\
\hline$\left\{\alpha_{21} \mathbf{u}_{34} \beta_{63}\right\}$ & $\left\{\alpha_{21} \alpha_{32} u_{63}\right\}$ & $\left\{\gamma_{21} \alpha_{65} \beta_{63}\right\}$ & $\left\{\alpha_{10} \alpha_{54} \beta_{63}\right\}$ & $\left\{\alpha_{65} \beta_{34} \alpha_{40}\right\}$ \\
\hline$\left\{\alpha_{21} \mathbf{u}_{34} \mathbf{u}_{63}\right\}$ & $\left\{\alpha_{21} \beta_{34} \alpha_{54}\right\}$ & $\left\{\mathbf{u}_{21} \beta_{34} \beta_{63}\right\}$ & $\left\{\alpha_{10} \alpha_{54} \mathbf{u}_{63}\right\}$ & $\left\{\alpha_{65} \alpha_{40} u_{34}\right\}$ \\
\hline$\left\{\alpha_{21} \alpha_{40} \alpha_{54}\right\}$ & $\left\{\alpha_{21} \beta_{34} \alpha_{65}\right\}$ & $\left\{\mathbf{u}_{21} \beta_{34} \mathbf{u}_{63}\right\}$ & $\left\{\alpha_{10} \alpha_{65} \beta_{63}\right\}$ & 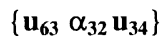 \\
\hline$\left\{\alpha_{21} \alpha_{40} \alpha_{65}\right\}$ & $\left\{\alpha_{21} \beta_{34} \beta_{63}\right\}$ & $\left\{\mathbf{u}_{21} \mathbf{u}_{34} \alpha_{54}\right\}$ & $\left\{\alpha_{10} \alpha_{65} \mathbf{u}_{63}\right\}$ & $\left\{u_{63} \alpha_{32} \alpha_{40}\right\}$ \\
\hline$\left\{\alpha_{21} \alpha_{40} \beta_{63}\right\}$ & $\left\{\alpha_{21} \beta_{34} \mathbf{u}_{63}\right\}$ & $\left\{u_{21} u_{34} \alpha_{65}\right\}$ & $\left\{\alpha_{10} \mathbf{u}_{63} \beta_{63}\right\}$ & $\left\{\begin{array}{lll}\mathbf{u}_{63} & \beta_{34} & \mathbf{u}_{34}\end{array}\right\}$ \\
\hline$\left\{\alpha_{10} \alpha_{32} \alpha_{65}\right\}$ & $\left\{\begin{array}{lll}\alpha_{21} & \alpha_{40} & u_{63}\end{array}\right\}$ & $\left\{\mathbf{u}_{21} \mathbf{u}_{34} \beta_{63}\right\}$ & $\left\{\alpha_{20} \alpha_{54} \alpha_{65}\right\}$ & $\left\{\begin{array}{lll}\mathbf{u}_{63} & \beta_{34} & \alpha_{40}\end{array}\right\}$ \\
\hline$\left\{\alpha_{10} \alpha_{32} \beta_{63}\right\}$ & $\left\{\beta_{21} \alpha_{32} \alpha_{54}\right\}$ & $\left\{\begin{array}{lll}\mathbf{u}_{21} & \mathbf{u}_{34} & \mathbf{u}_{63}\end{array}\right\}$ & $\left\{\alpha_{20} \alpha_{54} \beta_{63}\right\}$ & $\left\{\begin{array}{lll}\mathbf{u}_{63} & \alpha_{40} & u_{34}\end{array}\right\}$ \\
\hline$\left\{\alpha_{10} \alpha_{32} \mathbf{u}_{63}\right\}$ & $\left\{\beta_{21} \alpha_{32} \alpha_{65}\right\}$ & $\left\{\beta_{63} \alpha_{32} \beta_{34}\right\}$ & $\left\{\alpha_{20} \alpha_{54} \mathbf{u}_{63}\right\}$ & $\left\{\alpha_{32} \alpha_{54} \alpha_{65}\right\}$ \\
\hline$\left\{\alpha_{10} \beta_{34} \alpha_{54}\right\}$ & $\left\{\beta_{21} \alpha_{32} \beta_{63}\right\}$ & $\left\{\gamma_{21} \beta_{34} \beta_{63}\right\}$ & $\left\{\alpha_{20} \alpha_{65} \beta_{63}\right\}$ & $\left\{\alpha_{32} \alpha_{54} \beta_{63}\right\}$ \\
\hline$\left\{\alpha_{10} \beta_{34} \alpha_{65}\right\}$ & $\left\{\beta_{21} \alpha_{32} \mathbf{u}_{63}\right\}$ & $\left\{\gamma_{21} \beta_{34} \mathbf{u}_{63}\right\}$ & $\left\{\alpha_{20} \alpha_{65} \mathbf{u}_{63}\right\}$ & $\left\{\alpha_{32} \alpha_{54} \mathbf{u}_{63}\right\}$ \\
\hline$\left\{v_{21} \alpha_{32} \alpha_{65}\right\}$ & $\left\{\beta_{21} \beta_{34} \alpha_{54}\right\}$ & $\left\{\gamma_{21} \mathbf{u}_{34} \alpha_{54}\right\}$ & $\left\{\alpha_{20} \mathbf{u}_{63} \beta_{63}\right\}$ & $\left\{\alpha_{32} \alpha_{65} \beta_{63}\right\}$ \\
\hline$\left\{\begin{array}{lll}v_{21} & \alpha_{32} & \beta_{63}\end{array}\right\}$ & $\left\{\beta_{21} \beta_{34} \alpha_{65}\right\}$ & $\left\{\gamma_{21} \mathbf{u}_{34} \alpha_{65}\right\}$ & $\left\{\alpha_{21} \alpha_{54} \alpha_{65}\right\}$ & $\left\{\alpha_{32} \alpha_{65} u_{63}\right\}$ \\
\hline$\left\{\begin{array}{lll}\mathbf{v}_{21} & \alpha_{32} & \mathbf{u}_{63}\end{array}\right\}$ & $\left\{\beta_{21} \beta_{34} \beta_{63}\right\}$ & $\left\{\gamma_{21} \mathbf{u}_{34} \beta_{63}\right\}$ & $\left\{\beta_{21} \alpha_{54} \alpha_{65}\right\}$ & $\left\{\alpha_{32} u_{63} \beta_{63}\right\}$ \\
\hline$\left\{v_{21} \beta_{34} \alpha_{54}\right\}$ & $\left\{\beta_{21} \beta_{34} \mathbf{u}_{63}\right\}$ & $\left\{\beta_{63} \alpha_{32} \mathbf{u}_{34}\right\}$ & $\left\{\beta_{21} \alpha_{54} \beta_{63}\right\}$ & $\left\{\beta_{34} \alpha_{54} \alpha_{65}\right\}$ \\
\hline$\left\{v_{21} \beta_{34} \alpha_{65}\right\}$ & $\left\{\beta_{21} \mathbf{u}_{34} \alpha_{54}\right\}$ & $\left\{\beta_{63} \alpha_{32} \alpha_{40}\right\}$ & $\left\{\beta_{21} \alpha_{54} \mathbf{u}_{63}\right\}$ & $\left\{\beta_{34} \alpha_{54} \beta_{63}\right\}$ \\
\hline$\left\{\begin{array}{lll}\alpha_{10} & \alpha_{40} & \alpha_{65}\end{array}\right\}$ & $\left\{\beta_{21} \mathbf{u}_{34} \alpha_{65}\right\}$ & $\left\{\begin{array}{ll}\beta_{63} & \beta_{34} \mathbf{u}_{34}\end{array}\right\}$ & $\left\{\beta_{21} \alpha_{65} \beta_{63}\right\}$ & $\left\{\beta_{34} \alpha_{54} \mathbf{u}_{63}\right\}$ \\
\hline$\left\{\begin{array}{ll}\alpha_{10} & \alpha_{40} \beta_{63}\end{array}\right\}$ & $\left\{\beta_{21} \mathbf{u}_{34} \beta_{63}\right\}$ & $\left\{\begin{array}{lll}\beta_{63} & \beta_{34} & \alpha_{40}\end{array}\right\}$ & $\left\{\beta_{21} \alpha_{65} u_{63}\right\}$ & $\left\{\beta_{34} \alpha_{65} \beta_{63}\right\}$ \\
\hline$\left\{\begin{array}{lll}\alpha_{10} & \alpha_{40} & \mathbf{u}_{63}\end{array}\right\}$ & $\left\{\beta_{21} \mathbf{u}_{34} \mathbf{u}_{63}\right\}$ & $\left\{\begin{array}{lll}\beta_{63} & \alpha_{40} & u_{34}\end{array}\right\}$ & $\left\{\beta_{21} \mathbf{u}_{63} \beta_{63}\right\}$ & $\left\{\beta_{34} \alpha_{65} \mathbf{u}_{63}\right\}$ \\
\hline$\left\{\alpha_{20} \alpha_{32} \alpha_{54}\right\}$ & $\left\{\begin{array}{lll}\beta_{21} & \alpha_{40} & \alpha_{54}\end{array}\right\}$ & $\left\{\mathbf{u}_{63} \alpha_{32} \beta_{34}\right\}$ & $\left\{\gamma_{21} \alpha_{65} \mathbf{u}_{63}\right\}$ & $\left\{\beta_{34} \mathbf{u}_{63} \beta_{63}\right\}$ \\
\hline$\left\{\alpha_{21} \alpha_{54} \beta_{63}\right\}$ & $\left\{\beta_{21} \alpha_{40} \alpha_{65}\right\}$ & $\left\{\begin{array}{lll}\mathbf{u}_{21} & \alpha_{40} & \alpha_{54}\end{array}\right\}$ & $\left\{\gamma_{21} \mathbf{u}_{63} \beta_{63}\right\}$ & $\left\{u_{34} \alpha_{54} \alpha_{65}\right\}$ \\
\hline$\left\{\alpha_{21} \alpha_{54} \mathbf{u}_{63}\right\}$ & $\left\{\beta_{21} \alpha_{40} \beta_{63}\right\}$ & $\left\{u_{21} \alpha_{40} \alpha_{65}\right\}$ & $\left\{u_{21} \alpha_{54} \alpha_{65}\right\}$ & $\left\{u_{34} \alpha_{54} \beta_{63}\right\}$ \\
\hline$\left\{\alpha_{21} \alpha_{65} \beta_{63}\right\}$ & $\left\{\beta_{21} \alpha_{40} u_{63}\right\}$ & $\left\{u_{21} \alpha_{40} \beta_{63}\right\}$ & $\left\{u_{21} \alpha_{54} \beta_{63}\right\}$ & $\left\{\mathbf{u}_{34} \alpha_{54} \mathbf{u}_{63}\right\}$ \\
\hline$\left\{\alpha_{21} \alpha_{65} u_{63}\right\}$ & $\left\{\gamma_{21} \alpha_{32} \alpha_{54}\right\}$ & $\left\{\begin{array}{lll}\mathbf{u}_{21} & \alpha_{40} & \mathbf{u}_{63}\end{array}\right\}$ & $\left\{\mathbf{u}_{21} \alpha_{54} \mathbf{u}_{63}\right\}$ & $\left\{u_{34} \alpha_{65} \beta_{63}\right\}$ \\
\hline$\left\{\alpha_{21} \mathbf{u}_{63} \beta_{63}\right\}$ & $\left\{\gamma_{21} \alpha_{32} \alpha_{65}\right\}$ & $\left\{\mathbf{v}_{21} \alpha_{32} \alpha_{54}\right\}$ & $\left\{\mathbf{u}_{21} \alpha_{65} \beta_{63}\right\}$ & $\left\{\mathbf{u}_{34} \alpha_{65} \mathbf{u}_{63}\right\}$ \\
\hline$\left\{\alpha_{20} \alpha_{32} \alpha_{65}\right\}$ & $\left\{\begin{array}{lll}\gamma_{21} & \alpha_{32} & \beta_{63}\end{array}\right\}$ & $\left\{\alpha_{10} \beta_{34} \beta_{63}\right\}$ & $\left\{\mathbf{u}_{21} \alpha_{65} u_{63}\right\}$ & $\left\{u_{34} u_{63} \beta_{63}\right\}$ \\
\hline$\left\{\alpha_{20} \alpha_{32} \beta_{63}\right\}$ & $\left\{\begin{array}{lll}\gamma_{21} & \alpha_{32} & u_{63}\end{array}\right\}$ & $\left\{\alpha_{10} \beta_{34} \mathbf{u}_{63}\right\}$ & $\left\{\mathbf{u}_{21} \mathbf{u}_{63} \boldsymbol{\beta}_{63}\right\}$ & $\left\{\alpha_{40} \alpha_{54} \alpha_{65}\right\}$ \\
\hline$\left\{\begin{array}{lll}u_{20} & \alpha_{32} & \alpha_{63} \\
\alpha_{32} & u_{63}\end{array}\right\}$ & $\left\{\gamma_{21} \beta_{34} \alpha_{54}\right\}$ & $\left\{\alpha_{10} \mathbf{u}_{34} \alpha_{54}\right\}$ & $\left\{v_{21} \alpha_{54} \alpha_{65}\right\}$ & $\left\{\alpha_{40} \alpha_{54} \beta_{63}\right\}$ \\
\hline$\left\{\alpha_{20} \beta_{34} \alpha_{54}\right\}$ & $\left\{\gamma_{21} \beta_{34} \alpha_{65}\right\}$ & $\left\{\alpha_{10} \mathbf{u}_{34} \alpha_{65}\right\}$ & $\left\{\mathbf{v}_{21} \alpha_{54} \beta_{63}\right\}$ & $\left\{\alpha_{40} \alpha_{54} \mathbf{u}_{63}\right\}$ \\
\hline$\left\{\alpha_{20} \beta_{34} \alpha_{65}\right\}$ & $\left\{\gamma_{21} \mathbf{u}_{34} \mathbf{u}_{63}\right\}$ & $\left\{\alpha_{10} \mathbf{u}_{34} \beta_{63}\right\}$ & $\left\{\mathbf{v}_{21} \alpha_{54} \mathbf{u}_{63}\right\}$ & $\left\{\alpha_{40} \alpha_{65} \beta_{63}\right\}$ \\
\hline$\left\{\alpha_{20} \beta_{34} \beta_{63}\right\}$ & $\left\{\gamma_{21} \alpha_{40} \alpha_{54}\right\}$ & $\left\{\alpha_{10} \mathbf{u}_{34} \mathbf{u}_{63}\right\}$ & $\left\{v_{21} \alpha_{65} \beta_{63}\right\}$ & $\left\{\alpha_{40} \alpha_{65} \mathbf{u}_{63}\right\}$ \\
\hline$\left\{\alpha_{20} \beta_{34} \mathbf{u}_{63}\right\}$ & $\left\{\begin{array}{lll}\gamma_{21} & \alpha_{40} & \alpha_{65}\end{array}\right\}$ & $\left\{\begin{array}{ll}\alpha_{10} & \alpha_{40} \\
\alpha_{54}\end{array}\right\}$ & $\left\{\mathbf{v}_{21} \alpha_{65} u_{63}\right\}$ & $\left\{\alpha_{40} \mathbf{u}_{63} \beta_{63}\right\}$ \\
\hline$\left\{\alpha_{20} \mathbf{u}_{34} \alpha_{54}\right\}$ & $\left\{\gamma_{21} \alpha_{40} \beta_{63}\right\}$ & $\left\{v_{21} \beta_{34} \beta_{63}\right\}$ & $\left\{\mathbf{v}_{21} \mathbf{u}_{63} \beta_{63}\right\}$ & $\left\{\alpha_{54} \alpha_{65} \beta_{63}\right\}$ \\
\hline$\left\{\alpha_{20} u_{34} \alpha_{65}\right\}$ & $\left\{\begin{array}{lll}\gamma_{21} & \alpha_{40} & \mathbf{u}_{63}\end{array}\right\}$ & $\left\{\mathbf{v}_{21} \boldsymbol{\beta}_{34} \mathbf{u}_{63}\right\}$ & $\left\{\alpha_{54} \alpha_{32} \beta_{34}\right\}$ & $\left\{\alpha_{54} \alpha_{65} \mathbf{u}_{63}\right\}$ \\
\hline$\left\{\alpha_{20} \mathbf{u}_{34} \beta_{63}\right\}$ & $\left\{\mathbf{u}_{21} \alpha_{32} \alpha_{54}\right\}$ & $\left\{\mathbf{v}_{21} \mathbf{u}_{34} \alpha_{54}\right\}$ & $\left\{\alpha_{54} \alpha_{32} u_{34}\right\}$ & $\left\{\begin{array}{lll}\alpha_{54} & \mathbf{u}_{63} & \beta_{63}\end{array}\right\}$ \\
\hline$\left\{\alpha_{20} \mathbf{u}_{34} \mathbf{u}_{63}\right\}$ & $\left\{\mathbf{u}_{21} \alpha_{32} \alpha_{65}\right\}$ & $\left\{\mathbf{v}_{21} \mathbf{u}_{34} \alpha_{65}\right\}$ & $\left\{\alpha_{54} \alpha_{32} \alpha_{40}\right\}$ & $\left\{u_{63} \alpha_{65} \beta_{63}\right\}$ \\
\hline$\left\{\begin{array}{lll}\alpha_{20} & \alpha_{40} & \alpha_{54}\end{array}\right\}$ & $\left\{u_{21} \alpha_{32} \beta_{63}\right\}$ & $\left\{\begin{array}{lll}v_{21} & u_{34} & \beta_{63}\end{array}\right\}$ & $\left\{\begin{array}{lll}\alpha_{54} & \beta_{34} & \mathbf{u}_{34}\end{array}\right\}$ & \\
\hline$\left\{\begin{array}{lll}\alpha_{20} & \alpha_{40} & \alpha_{65}\end{array}\right\}$ & $\left\{\begin{array}{lll}\mathbf{u}_{21} & \alpha_{32} & \mathbf{u}_{63}\end{array}\right\}$ & $\left\{\begin{array}{lll}\mathbf{v}_{21} & \mathbf{u}_{34} & \mathbf{u}_{63}\end{array}\right\}$ & $\left\{\alpha_{54} \beta_{34} \alpha_{40}\right\}$ & \\
\hline$\left\{\begin{array}{lll}\alpha_{20} & \alpha_{40} & \beta_{63}\end{array}\right\}$ & $\left\{\mathbf{u}_{21} \beta_{34} \alpha_{54}\right\}$ & $\left\{\mathbf{v}_{21} \alpha_{40} \alpha_{54}\right\}$ & $\left\{\begin{array}{llll}\alpha_{54} & \alpha_{40} & \mathbf{u}_{34}\end{array}\right\}$ & \\
\hline$\left\{\begin{array}{lll}\alpha_{20} & \alpha_{40} & \mathbf{u}_{63}\end{array}\right\}$ & $\left\{\mathbf{u}_{21} \beta_{34} \alpha_{65}\right\}$ & $\left\{v_{21} \alpha_{40} \alpha_{65}\right\}$ & $\left\{\alpha_{65} \alpha_{32} \beta_{34}\right\}$ & \\
\hline
\end{tabular}

cinématiques, nous avons développé une autre méthode de présentation de l'ensemble des jeux qui permet de :

- répartir les $\left(\mathrm{r}^{\prime \prime}+\mathrm{m}\right)$ inconnues cinématiques en $x$ ensembles $\mathrm{E}_{\mathrm{k}}(\mathrm{k}: 1 \ldots x)$;

- former tout jeu du tableau 3 à partir d'un choix guidé de $m$ paramètres dans ces ensembles.

Cette méthode est fondée sur la constatation, dans l'ensemble des jeux, que certains paramètres sont interchangeables. Pour mettre en évidence cette constatation, nous allons présenter deux exemples traitant chacun, d'une façon isolée, un sous-ensemble de jeux extraits du tableau 3. $\mathbf{1}^{\mathrm{er}}$ exemple : considérons un premier sous-ensemble composé des 7 jeux suivants

$$
\begin{aligned}
&\left\{\alpha_{10} \alpha_{32} \alpha_{54}\right\}\left\{\alpha_{20} \alpha_{32} \alpha_{54}\right\}\left\{\alpha_{21} \alpha_{32} \alpha_{54}\right\}\left\{\beta_{21} \alpha_{32} \alpha_{54}\right\} \\
&\left\{\gamma_{21} \alpha_{32} \alpha_{54}\right\}\left\{\mathbf{u}_{21} \alpha_{32} \alpha_{54}\right\}\left\{\mathbf{v}_{21} \alpha_{32} \alpha_{54}\right\} .
\end{aligned}
$$

Dans ces 7 jeux, nous constatons que $\alpha_{32}$ et $\alpha_{54}$ sont fixes et le paramètre qui change est l'un parmi l'ensemble $\left\{\alpha_{10} \alpha_{20} \alpha_{21} \beta_{21} \gamma_{21} \mathbf{u}_{21} \mathbf{v}_{21}\right\}$. Nous pouvons alors répartir les inconnues participant dans ces jeux en deux ensembles $\mathrm{E}_{1}:\left\{\alpha_{32} \alpha_{54}\right\}$ et $\mathrm{E}_{2}:\left\{\alpha_{10} \alpha_{20} \alpha_{21} \beta_{21} \gamma_{21} \mathbf{u}_{21} \mathbf{v}_{21}\right\}$. Chacun des 7 jeux peut être formé en choisissant arbitrairement deux paramètres dans $\mathrm{E}_{1}$ et un paramètre dans $\mathrm{E}_{2}$. 
$\mathbf{2}^{\mathbf{e}}$ exemple : considérons un deuxième sous-ensemble composé des 7 jeux suivants

$$
\begin{array}{r}
\left\{\alpha_{32} \alpha_{54} \alpha_{65}\right\}\left\{\alpha_{32} \alpha_{54} \beta_{63}\right\}\left\{\alpha_{32} \alpha_{65} \beta_{63}\right\}\left\{\beta_{34} \alpha_{54} \alpha_{65}\right\} \\
\left\{\beta_{34} \alpha_{54} \beta_{63}\right\}\left\{\beta_{34} \alpha_{65} \beta_{63}\right\}\left\{\alpha_{54} \alpha_{65} \beta_{63}\right\} .
\end{array}
$$

Dans les 6 premiers jeux nous constatons que :

- pour un jeu qui contient $\alpha_{32}$ il existe son analogue qui contient $\beta_{34}$,

- deux parmi les inconnues $\alpha_{54}, \alpha_{65}$ et $\beta_{63}$ sont présentes dans chaque jeu.

Le dernier jeu contient les 3 paramètres $\alpha_{54}, \alpha_{65}$ et $\beta_{63}$.

Nous pouvons répartir les inconnues participant dans ces jeux en deux ensembles $\mathrm{E}_{1}:\left\{\alpha_{32} \beta_{34}\right\}$ et $\mathrm{E}_{2}$ : $\left\{\alpha_{54} \alpha_{65} \beta_{63}\right\}$. Pour obtenir un des 7 jeux nous devons choisir

- un paramètre de $\mathrm{E}_{1}$ et deux paramètres de $\mathrm{E}_{2}$

- ou trois paramètres de $\mathrm{E}_{2}$.

\subsubsection{Relation d'équivalence pour la répartition des inconnues en ensembles}

Nous remarquons à partir des deux exemples précédents, qu'un choix guidé des paramètres dans les ensembles $\mathrm{E}_{\mathrm{k}}$ des inconnues, permet de reconstruire une partie ou tous les jeux des paramètres cinématiques indépendants. Mais selon le deuxième exemple, ce choix peut ne pas être unique. En effet, pour une répartition déterminée, il peut exister plusieurs possibilités de choix pour retrouver l'ensemble complet des jeux. Une possibilité $\mathrm{t}$ de choix correspond au choix arbitraire de $n_{\mathrm{tk}}$ paramètres dans chaque ensemble $\mathrm{E}_{\mathrm{k}}$ avec $\sum_{\mathrm{k}=1}^{\mathrm{x}} \mathrm{n}_{\mathrm{tk}}=\mathrm{m}$.

Nous avons vu aussi que l'interchangeabilité entre les inconnues constitue la condition nécessaire pour la formation d'un ensemble $\mathrm{E}_{\mathrm{k}}$. En effet, si nous effectuons un remplacement réciproque entre deux inconnues de $\mathrm{E}_{\mathrm{k}}$ dans les jeux où figure l'une d'elles, nous obtenons les jeux où figure l'autre inconnue. C'est ce que nous pouvons vérifier facilement dans les deux exemples précédents. Nous pouvons donc affirmer qu'il existe une relation d'équivalence entre les inconnues d'un même ensemble $\mathrm{E}_{\mathrm{k}}$.

Considérons $\mathrm{J}(\mathbf{u})$ et $\mathrm{J}(\mathbf{v})$ les deux sous-ensembles où figurent tous les jeux contenant respectivement l'inconnue cinématique $\mathbf{u}$ et l'inconnue cinématique $\mathbf{v}$.

Pour pouvoir choisir arbitrairement $n_{j k}$ inconnues dans un ensemble $E_{k}$, il faut satisfaire la condition suivante :

$\forall \mathbf{v} \in \mathrm{E}_{\mathrm{k}}, \forall \mathbf{u} \in \mathrm{E}_{\mathrm{k}}$, en remplaçant dans les jeux de $\mathrm{J}(\mathbf{v})$, $\mathbf{v}$ par $\mathbf{u}$ et $\mathbf{u}$ par $\mathbf{v}$ on obtient $J(\mathbf{u})$, et réciproquement.

Cette condition définit une relation d'équivalence dont les classes $E_{k}$ engendrent une partition des $\left(r^{\prime \prime}+m\right)$ inconnues cinématiques.

Pour vérifier si deux inconnues quelconques $\mathbf{u}$ et $\mathbf{v}$ appartiennent au même ensemble, nous devons procéder à une comparaison entre les deux sous-ensembles des jeux $\mathrm{J}(\mathbf{u})$ et $\mathrm{J}(\mathbf{v})$. Cette comparaison est réalisée en trois étapes : $-1^{\text {re }}$ étape : la formation des sous-ensembles $J(\mathbf{u})$ et $\mathrm{J}(\mathbf{v})$ à partir de l'ensemble des jeux ;

- $2^{\mathrm{e}}$ étape : la vérification de l'égalité des cardinaux $\operatorname{Card}(\mathrm{J}(\mathbf{u}))=\operatorname{Card}(\mathrm{J}(\mathbf{v}))$;

- $3^{\mathrm{e}}$ étape : si les cardinaux sont égaux, nous passons à la comparaison des constitutions des jeux de $\mathrm{J}(\mathbf{u})$ et $\mathrm{J}(\mathbf{v})$ après la permutation dans l'un d'eux entre $\mathbf{u}$ et $\mathbf{v}$ en vue de vérifier la relation d'équivalence ci-dessus.

Prenons l'exemple des deux inconnues $\alpha_{10}$ et $\alpha_{20}$ du mécanisme de l'essuie-glace. À partir de la liste du tableau 3, nous formons les deux sous-ensembles $\mathrm{J}\left(\alpha_{10}\right)$ et $\mathrm{J}\left(\alpha_{20}\right)$ du tableau 4.

Les cardinaux de ces deux sous-ensembles étant égaux, $\operatorname{Card}\left(\mathrm{J}\left(\alpha_{10}\right)\right)=\operatorname{Card}\left(\mathrm{J}\left(\alpha_{20}\right)\right)=22$, nous passons à la troisième étape en permutant dans $\mathrm{J}\left(\alpha_{10}\right)$ entre $\alpha_{10}$ et $\alpha_{20}$ pour obtenir un nouveau sous-ensemble $\mathrm{J}^{\prime}\left(\alpha_{10}\right)$. Dans cet exemple où nous avons présenté les jeux et les paramètres d'une façon ordonnée, nous pouvons constater facilement que $\mathrm{J}^{\prime}\left(\alpha_{10}\right)=\mathrm{J}\left(\alpha_{20}\right)$, ainsi les deux inconnues $\alpha_{10}$ et $\alpha_{20}$ appartiennent au même ensemble $\mathrm{E}_{\mathrm{k}}$. Mais d'une façon générale, la mise en ouvre informatique de la vérification de l'égalité $\mathrm{J}^{\prime}(\mathbf{u})=\mathrm{J}(\mathbf{v})$ est assez lourde, car pour tout jeu de $\mathrm{J}^{\prime}(\mathbf{u})$, il va falloir chercher son similaire dans les jeux de $J(\mathbf{v})$. Une opération qui se complique plus si le cardinal de $\mathrm{J}^{\prime}(\mathrm{u})$ est élevé ou/et le degré de mobilité est important.

Pour simplifier cette opération, nous représentons d'une façon unique, chaque jeu dans chaque sousensemble par un nombre entier. Ainsi nous transformons les sous-ensembles $\mathrm{J}^{\prime}(\mathbf{u})$ et $\mathrm{J}(\mathbf{v})$ en sous-ensembles des nombres entiers et nous rendons la comparaison plus rapide.

\subsubsection{Transformation d'un sous-ensemble des jeux en sous-ensemble des nombres entiers}

Chaque sous-ensemble $\mathrm{J}(\mathbf{u})$ est représenté par une matrice $\mathrm{M}(\mathrm{J}(\mathbf{u}))$ de dimensions $\left(\operatorname{Card}(\mathrm{J}(\mathbf{u})), \mathrm{r}^{\prime \prime}+\mathrm{m}\right)$. Les inconnues numérotées de 1 à $\left(\mathrm{r}^{\prime \prime}+\mathrm{m}\right)$, forment les colonnes de $\mathrm{M}(\mathrm{J}(\mathbf{u}))$. Chaque ligne i représente un jeu i de $\mathrm{J}(\mathbf{u})$. Chaque coefficient $\mu_{\text {iq }}$ de la matrice $\mathrm{M}(\mathrm{J}(\mathbf{u}))$ est égal à 0 ou 1 selon que l'inconnue numéro q figure dans le jeu i ou non.

Le nombre entier unique, noté $\mathrm{N}_{\mathrm{i}}$, associé à chaque ligne i, donc à chaque jeu, est calculé de la façon suivante

$$
\mathrm{N}_{\mathrm{i}}=\sum_{\mathrm{q}=1}^{\mathrm{r}^{\prime \prime}+\mathrm{m}} \mu_{\mathrm{iq}} \cdot \mathrm{q} \cdot 10^{\lambda\left(\sum_{\mathrm{k}=1}^{\mathrm{j}} \mu_{\mathrm{ik}}-1\right)}
$$

le facteur $\lambda$ représente le nombre de chiffres réservés pour chaque inconnue. Si le nombre des inconnues $\left(\mathrm{r}^{\prime \prime}+\mathrm{m}\right)$ ne dépasse pas 99 , nous pouvons prendre $\lambda=2$, sinon nous prendrons $\lambda=3$ ou 4 etc.

Appliquons ce processus de numérotation sur le sousensemble $\mathrm{J}^{\prime}\left(\alpha_{10}\right)$ du mécanisme d'essuie-glace. Les inconnues cinématiques sont numérotées selon leur ordre dans les vecteurs $\left\{\mathbf{V}_{1}^{\prime}\right\}$ et $\{\mathbf{W}\}$ (cf. Sect. 4.2.1) 
Tableau 4. Sous-ensembles $\mathrm{J}\left(\alpha_{10}\right)$ et $\mathrm{J}\left(\alpha_{20}\right)$.

\begin{tabular}{|c|c|c|}
\hline & $\mathrm{J}\left(\alpha_{10}\right)$ & $\mathrm{J}\left(\alpha_{20}\right)$ \\
\hline 1 & $\left\{\begin{array}{lll}\alpha_{10} & \alpha_{32} & \alpha_{54}\end{array}\right\}$ & $\left\{\begin{array}{lll}\alpha_{20} & \alpha_{32} & \alpha_{54}\end{array}\right\}$ \\
\hline 2 & $\left\{\begin{array}{lll}\alpha_{10} & \alpha_{32} & \alpha_{65}\end{array}\right\}$ & $\left\{\begin{array}{lll}\alpha_{20} & \alpha_{32} & \alpha_{65}\end{array}\right\}$ \\
\hline 3 & $\left\{\begin{array}{lll}\alpha_{10} & \alpha_{32} & \beta_{63}\end{array}\right\}$ & $\left\{\begin{array}{lll}\alpha_{20} & \alpha_{32} & \beta_{63}\end{array}\right\}$ \\
\hline 4 & $\left\{\begin{array}{lll}\alpha_{10} & \alpha_{32} & \mathbf{u}_{63}\end{array}\right\}$ & $\left\{\begin{array}{lll}\alpha_{20} & \alpha_{32} & \mathbf{u}_{63}\end{array}\right\}$ \\
\hline 5 & $\left\{\begin{array}{lll}\alpha_{10} & \beta_{34} & \alpha_{54}\end{array}\right\}$ & $\left\{\begin{array}{lll}\alpha_{20} & \beta_{34} & \alpha_{54}\end{array}\right\}$ \\
\hline 6 & $\left\{\begin{array}{llll}\alpha_{10} & \beta_{34} & \alpha_{65}\end{array}\right\}$ & $\left\{\begin{array}{lll}\alpha_{20} & \beta_{34} & \alpha_{65}\end{array}\right\}$ \\
\hline 7 & $\left\{\begin{array}{lll}\alpha_{10} & \beta_{34} & \beta_{63}\end{array}\right\}$ & $\left\{\alpha_{20} \beta_{34} \beta_{63}\right\}$ \\
\hline 8 & $\left\{\begin{array}{lll}\alpha_{10} & \beta_{34} & \mathbf{u}_{63}\end{array}\right\}$ & $\left\{\begin{array}{lll}\alpha_{20} & \beta_{34} & \mathbf{u}_{63}\end{array}\right\}$ \\
\hline 9 & $\left\{\begin{array}{lll}\alpha_{10} & \alpha_{40} & \alpha_{54}\end{array}\right\}$ & $\left\{\begin{array}{lll}\alpha_{20} & \alpha_{40} & \alpha_{54}\end{array}\right\}$ \\
\hline 10 & $\left\{\begin{array}{lll}\alpha_{10} & \alpha_{40} & \alpha_{65}\end{array}\right\}$ & $\left\{\begin{array}{lll}\alpha_{20} & \alpha_{40} & \alpha_{65}\end{array}\right\}$ \\
\hline 11 & $\left\{\begin{array}{lll}\alpha_{10} & \alpha_{40} & \beta_{63}\end{array}\right\}$ & $\left\{\begin{array}{lll}\alpha_{20} & \alpha_{40} & \beta_{63}\end{array}\right\}$ \\
\hline 12 & $\left\{\begin{array}{lll}\alpha_{10} & \alpha_{40} & \mathbf{u}_{63}\end{array}\right\}$ & $\left\{\begin{array}{lll}\alpha_{20} & \alpha_{40} & \mathbf{u}_{63}\end{array}\right\}$ \\
\hline 13 & $\left\{\begin{array}{lll}\alpha_{10} & \alpha_{54} & \alpha_{65}\end{array}\right\}$ & $\left\{\begin{array}{lll}\alpha_{20} & \alpha_{54} & \alpha_{65}\end{array}\right\}$ \\
\hline 14 & $\left\{\begin{array}{lll}\alpha_{10} & \alpha_{54} & \beta_{63}\end{array}\right\}$ & $\left\{\begin{array}{lll}\alpha_{20} & \alpha_{54} & \beta_{63}\end{array}\right\}$ \\
\hline 15 & $\left\{\begin{array}{lll}\alpha_{10} & \alpha_{54} & \mathbf{u}_{63}\end{array}\right\}$ & $\left\{\begin{array}{lll}\alpha_{20} & \alpha_{54} & \mathbf{u}_{63}\end{array}\right\}$ \\
\hline 16 & $\left\{\begin{array}{lll}\alpha_{10} & \alpha_{65} & \beta_{63}\end{array}\right\}$ & $\left\{\begin{array}{lll}\alpha_{20} & \alpha_{65} & \beta_{63}\end{array}\right\}$ \\
\hline 17 & $\left\{\begin{array}{lll}\alpha_{10} & \alpha_{65} & \mathbf{u}_{63}\end{array}\right\}$ & $\left\{\begin{array}{lll}\alpha_{20} & \alpha_{65} & \mathbf{u}_{63}\end{array}\right\}$ \\
\hline 18 & $\left\{\begin{array}{lll}\alpha_{10} & \mathbf{u}_{63} & \beta_{63}\end{array}\right\}$ & $\left\{\begin{array}{lll}\alpha_{20} & \mathbf{u}_{63} & \beta_{63}\end{array}\right\}$ \\
\hline 19 & $\left\{\begin{array}{lll}\alpha_{10} & \mathbf{u}_{34} & \alpha_{54}\end{array}\right\}$ & $\left\{\begin{array}{lll}\alpha_{20} & \mathbf{u}_{34} & \alpha_{54}\end{array}\right\}$ \\
\hline 20 & $\left\{\begin{array}{lll}\alpha_{10} & \mathbf{u}_{34} & \alpha_{65}\end{array}\right\}$ & $\left\{\begin{array}{lll}\alpha_{20} & \mathbf{u}_{34} & \alpha_{65}\end{array}\right\}$ \\
\hline 21 & $\left\{\begin{array}{lll}\alpha_{10} & \mathbf{u}_{34} & \beta_{63}\end{array}\right\}$ & $\left\{\begin{array}{lll}\alpha_{20} & \mathbf{u}_{34} & \beta_{63}\end{array}\right\}$ \\
\hline 22 & $\left\{\begin{array}{lll}\alpha_{10} & \mathbf{u}_{34} & \mathbf{u}_{63}\end{array}\right\}$ & $\left\{\begin{array}{lll}\alpha_{20} & \mathbf{u}_{34} & \mathbf{u}_{63}\end{array}\right\}$ \\
\hline
\end{tabular}

Tableau 5. Matrice $\mathrm{M}\left(\mathrm{J}^{\prime}\left(\alpha_{10}\right)\right)$ et des nombres $\mathrm{N}_{\mathrm{i}}$ correspondant.

\begin{tabular}{ccccccccccccccccc}
\hline & 1 & 2 & 3 & 4 & 5 & 6 & 7 & 8 & 9 & 10 & 11 & 12 & 13 & 14 & 15 & $\mathrm{~N}_{\mathrm{i}}$ \\
& $\beta_{34}$ & $\alpha_{65}$ & $\alpha_{20}$ & $\alpha_{21}$ & $\beta_{21}$ & $\alpha_{40}$ & $\alpha_{32}$ & $\gamma_{21}$ & $\alpha_{54}$ & $\alpha_{10}$ & $\beta_{63}$ & $\mathbf{u}_{21}$ & $\mathbf{v}_{21}$ & $\mathbf{u}_{63}$ & $\mathbf{u}_{34}$ & \\
\hline 1 & 0 & 0 & 1 & 0 & 0 & 0 & 1 & 0 & 1 & 0 & 0 & 0 & 0 & 0 & 0 & 90703 \\
2 & 0 & 1 & 1 & 0 & 0 & 0 & 1 & 0 & 0 & 0 & 0 & 0 & 0 & 0 & 0 & 70302 \\
3 & 0 & 0 & 1 & 0 & 0 & 0 & 1 & 0 & 0 & 0 & 1 & 0 & 0 & 0 & 0 & 110703 \\
4 & 0 & 0 & 1 & 0 & 0 & 0 & 1 & 0 & 0 & 0 & 0 & 0 & 0 & 1 & 0 & 140703 \\
5 & 1 & 0 & 1 & 0 & 0 & 0 & 0 & 0 & 1 & 0 & 0 & 0 & 0 & 0 & 0 & 90301 \\
6 & 1 & 1 & 1 & 0 & 0 & 0 & 0 & 0 & 0 & 0 & 0 & 0 & 0 & 0 & 0 & 30201 \\
7 & 1 & 0 & 1 & 0 & 0 & 0 & 0 & 0 & 0 & 0 & 1 & 0 & 0 & 0 & 0 & 110301 \\
8 & 1 & 0 & 1 & 0 & 0 & 0 & 0 & 0 & 0 & 0 & 0 & 0 & 0 & 1 & 0 & 140301 \\
9 & 0 & 0 & 1 & 0 & 0 & 1 & 0 & 0 & 1 & 0 & 0 & 0 & 0 & 0 & 0 & 90603 \\
10 & 0 & 1 & 1 & 0 & 0 & 1 & 0 & 0 & 0 & 0 & 0 & 0 & 0 & 0 & 0 & 60302 \\
11 & 0 & 0 & 1 & 0 & 0 & 1 & 0 & 0 & 0 & 0 & 1 & 0 & 0 & 0 & 0 & 110603 \\
12 & 0 & 0 & 1 & 0 & 0 & 1 & 0 & 0 & 0 & 0 & 0 & 0 & 0 & 1 & 0 & 140603 \\
13 & 0 & 1 & 1 & 0 & 0 & 0 & 0 & 0 & 1 & 0 & 0 & 0 & 0 & 0 & 0 & 60302 \\
14 & 0 & 0 & 1 & 0 & 0 & 0 & 0 & 0 & 1 & 0 & 1 & 0 & 0 & 0 & 0 & 110903 \\
15 & 0 & 0 & 1 & 0 & 0 & 0 & 0 & 0 & 1 & 0 & 0 & 0 & 0 & 1 & 0 & 140903 \\
16 & 0 & 1 & 1 & 0 & 0 & 0 & 0 & 0 & 0 & 0 & 1 & 0 & 0 & 0 & 0 & 110302 \\
17 & 0 & 1 & 1 & 0 & 0 & 0 & 0 & 0 & 0 & 0 & 0 & 0 & 0 & 1 & 0 & 140302 \\
18 & 0 & 0 & 1 & 0 & 0 & 0 & 0 & 0 & 0 & 0 & 1 & 0 & 0 & 1 & 0 & 141103 \\
19 & 0 & 0 & 1 & 0 & 0 & 0 & 0 & 0 & 1 & 0 & 0 & 0 & 0 & 0 & 1 & 150903 \\
20 & 0 & 1 & 1 & 0 & 0 & 0 & 0 & 0 & 0 & 0 & 0 & 0 & 0 & 0 & 1 & 150302 \\
21 & 0 & 0 & 1 & 0 & 0 & 0 & 0 & 0 & 0 & 0 & 1 & 0 & 0 & 0 & 1 & 151103 \\
22 & 0 & 0 & 1 & 0 & 0 & 0 & 0 & 0 & 0 & 0 & 0 & 0 & 0 & 1 & 1 & 151403 \\
\hline
\end{tabular}


Tableau 6. Possibilité de choix des paramètres cinématiques indépendants.

\begin{tabular}{cccc}
\cline { 2 - 4 } & $\mathrm{E}_{1}$ & $\mathrm{E}_{2}$ & $\mathrm{E}_{3}$ \\
\hline Possibilité 1 & 1 & 1 & 1 \\
Possibilité 2 & 0 & 2 & 1 \\
Possibilité 3 & 0 & 1 & 2 \\
Possibilité 4 & 1 & 0 & 2 \\
Possibilité 5 & 0 & 0 & 3 \\
\hline
\end{tabular}

$1: \beta_{34}, 2: \alpha_{65}, 3: \alpha_{20}, 4: \alpha_{21}, 5: \beta_{21}, 6: \alpha_{40}, 7: \alpha_{32}$, $8: \gamma_{21}, 9: \alpha_{54}, 10: \alpha_{10}, 11: \beta_{63}, 12: \mathbf{u}_{21}, 13: \mathbf{v}_{21}$, $14: \mathbf{u}_{63}, 15: \mathbf{u}_{34}$. Nous avons 15 inconnues, nous prendrons $\lambda=2$.

La matrice $\mathrm{M}\left(\mathrm{J}^{\prime}\left(\alpha_{10}\right)\right)$ est donnée par le tableau 5. Les nombres associés aux trois premiers jeux du tableau 5 sont calculés comme suit :

$$
\begin{aligned}
\mathrm{N}_{1} & =1 \cdot 3 \cdot 10^{2(1-1)}+1 \cdot 7 \cdot 10^{2(2-1)}+1 \cdot 9 \cdot 10^{2(3-1)} \\
& =90703 \\
\mathrm{~N}_{2} & =1 \cdot 2 \cdot 10^{2(1-1)}+1 \cdot 3 \cdot 10^{2(2-1)}+1 \cdot 7 \cdot 10^{2(3-1)} \\
& =70302 \\
\mathrm{~N}_{3} & =1 \cdot 3 \cdot 10^{2(1-1)}+1 \cdot 7 \cdot 10^{2(2-1)}+1 \cdot 11 \cdot 10^{2(3-1)} \\
& =110703
\end{aligned}
$$

Ainsi nous pouvons lire que le jeu 1 est constitué des paramètres 9,7 et 3 , le jeu 2 est constitué des paramètres 7,3 et 2 et ainsi de suite.

Nous effectuons la même chose pour $\mathrm{J}\left(\alpha_{20}\right)$ et nous obtenons les 22 numéros correspondants. La vérification de l'égalité $\mathrm{J}^{\prime}\left(\alpha_{10}\right)=\mathrm{J}\left(\alpha_{20}\right)$ est rendu ainsi très facile puisqu'il s'agira de chercher pour chaque numéro dans $\mathrm{J}^{\prime}\left(\alpha_{10}\right)$ son analogue dans $\mathrm{J}\left(\alpha_{20}\right)$.

\subsubsection{Processus d'obtention des ensembles $E_{k}$}

Nous pouvons maintenant énoncer le processus d'obtention des ensembles $\mathrm{E}_{\mathrm{k}}$ comme suit :

Après l'obtention des tous les jeux selon le processus exposé à la section 4.2.1,

- Pour chaque inconnue $\mathbf{u}$, former le sous-ensemble des jeux $\mathrm{J}(\mathbf{u})$;

- Répéter jusqu'à épuisement des inconnues :

Pour chaque inconnue $\mathbf{u}$ qui n'est pas encore affecté à un ensemble $\mathrm{E}_{\mathrm{k}}$ :

- affecter u à un nouvel ensemble $\mathrm{E}_{\mathrm{k} 0}$

- pour chaque inconnue $\mathbf{v}$ qui n'est pas encore affectée à un ensemble $\mathrm{E}_{\mathrm{k}}$ : si $\operatorname{Card}(\mathrm{J}(\mathbf{u}))=\operatorname{Card}(\mathrm{J}(\mathbf{v}))$ alors

- remplacer dans $J(\mathbf{u})$, u par $\mathbf{v}$ et $\mathbf{v}$ par $\mathbf{u}$ pour obtenir $\mathrm{J}^{\prime}(\mathbf{u})$

- calculer les nombres $\mathrm{N}_{i}$ dans chaque sousensemble

- si les $\mathrm{N}_{i}$ des deux sous ensembles $\mathrm{J}^{\prime}(\mathbf{u})$ et $\mathrm{J}(\mathbf{v})$ sont les mêmes alors $\mathbf{v} \in \mathrm{E}_{\mathrm{k} 0}$.
L'application de ce processus au mécanisme d'essuieglace nous donne les résultats suivants :

- ensemble $\mathrm{E}_{1}=\left\{\alpha_{10}, \alpha_{20}, \alpha_{21}, \beta_{21}, \gamma_{21}, \mathbf{u}_{21}, \mathbf{v}_{21}\right\} ;$

- ensemble $\mathrm{E}_{2}=\left\{\alpha_{32}, \beta_{34}, \mathbf{u}_{34}, \alpha_{40}\right\}$;

- ensemble $\mathrm{E}_{3}=\left\{\alpha_{54}, \alpha_{65}, \beta_{63}, \mathbf{u}_{63}\right\}$.

\subsubsection{Détermination des $n_{\mathrm{tk}}$}

Une fois les ensembles $E_{k}$ sont formés, l'opération d'obtention des $n_{\text {tk }}$ est d'une grande simplicité. Cette opération est réalisée grâce à un tableau dont les colonnes sont les ensembles $\mathrm{E}_{\mathrm{k}}$ et les lignes sont les différentes possibilités t de répartition des $\mathrm{n}_{\mathrm{tk}}$. Si le nombre des colonnes est fixé, la construction des lignes se fait progressivement. Elle est réalisée en parcourant l'ensemble des jeux un à un et en marquant dans l'intersection ligne-colonne le nombre des inconnues de l'ensemble $\mathrm{E}_{\mathrm{k}}$ contenues dans le jeu. Une ligne ne sera gardée que si elle est différente de toutes les lignes précédentes.

Voyons l'application de cette opération sur le mécanisme d'essuie-glace. Nous formons une matrice à trois colonnes selon le nombre des ensembles $\mathrm{E}_{\mathrm{k}}$ trouvés. L'examen des intersections inconnues $/ \mathrm{E}_{\mathrm{k}} \mathrm{du}$ premier jeu $\left\{\alpha_{10} \alpha_{32} \alpha_{54}\right\}$ du tableau 3 nous donne

\begin{tabular}{|c|c|c|}
\hline $\mathrm{E}_{1}$ & $\mathrm{E}_{2}$ & $\mathrm{E}_{3}$ \\
\hline 1 & 1 & 1 \\
\hline
\end{tabular}

Cette première ligne construite des $\mathrm{n}_{1 \mathrm{k}}$ constitue la première possibilité de choix des paramètres. L'examen du deuxième jeu $\left\{\alpha_{10} \alpha_{32} \alpha_{65}\right\}$ donne une ligne identique qui ne sera pas retenue car représentant la même possibilité 1 détectée. Cette opération effectuée pour les 206 jeux nous dévoile les 5 possibilités de choix du tableau 6 .

Finalement un jeu parmi les 206 jeux de paramètres cinématiques indépendants du tableau 3 est obtenu en choisissant 3 paramètres dans les 3 ensembles $\mathrm{E}_{1}, \mathrm{E}_{2}$ et $\mathrm{E}_{3}$ selon une des 5 possibilités du tableau 6 .

\section{Conclusion}

La méthode présentée d'analyse cinématique des mécanismes permet systématiquement à partir des seules données en liaisons de :

- détecter un jeu de cycles indépendants;

- mettre en équation le système;

- déterminer les degrés de mobilité et d'hyperstaticité du mécanisme;

- dégager les vitesses nulles;

- fournir tous les jeux des paramètres cinématiques indépendants. 
L'obtention systématique de ces résultats nous semble très bénéfique dans une phase d'avant projet de conception d'un mécanisme ou dans une opération d'expertise d'un mécanisme existant. Dans ces deux cas, le besoin intense d'une aide à la décision est assez rempli par les résultats obtenus. Cependant, nous devons signaler que ces résultats sont relatifs à une configuration donnée du mécanisme mais la méthode reste valable pour n'importe quelle configuration d'étude choisie.

\section{Annexe}

Les liaisons sont définies dans un repère local $R_{\text {local }}$ qui favorise l'écriture du torseur cinématique sous une forme canonique. D'une façon générale $\mathrm{R}_{\text {local }}$ est formé par :

- l'axe u, qui représente particulièrement la direction de la liaison dans le cas des liaisons à une direction (pivot, glissière, hélicoïdale, pivot glissant et linéaire rectiligne);

- l'axe v, qui représente particulièrement la normale au contact dans le cas des liaisons à une normale (appui ponctuel, linéaire rectiligne et appui plan);

- l'axe $\mathbf{w}$, qui vérifie la relation vectorielle $\mathbf{w}=\mathbf{u} \wedge \mathbf{v}$;

- l'origine $\mathrm{O}^{\prime}$ qui représente le centre de la liaison. Ce centre est défini dans le repère global $(\mathrm{O}, \mathbf{X}, \mathbf{Y}, \mathbf{Z})$ $\operatorname{par} \mathbf{O} \mathbf{O}^{\prime}=\left(\begin{array}{l}x \\ y \\ z\end{array}\right)$.

Dans ces conditions la matrice $\left[\mathbf{m}_{\mathrm{p}}\right]$ de passage du repère local $\left(\mathrm{O}^{\prime}, \mathbf{u}, \mathbf{v}, \mathbf{w}\right)$ au repère global $(\mathrm{O}, \mathbf{X}, \mathbf{Y}, \mathbf{Z})$ est définie par

$$
\left[\mathbf{m}_{\mathrm{p}}\right]=\left[\begin{array}{lll}
a_{1} & a_{2} & a_{3} \\
b_{1} & b_{2} & b_{3} \\
c_{1} & c_{2} & c_{3}
\end{array}\right]
$$

La matrice $\left[\mathbf{m}_{\mathrm{t}}\right]$ de transport du torseur du point $\mathrm{O}^{\prime}$ au point $\mathrm{O}$ est définie par

$$
\left[\mathbf{m}_{\mathrm{t}}\right]=\left[\begin{array}{ccc}
0 & -z & y \\
z & 0 & -x \\
-y & x & 0
\end{array}\right]
$$

Compte tenu de ces définitions, le torseur cinématique s'écrit selon la relation (11) s'écrit

$$
\begin{aligned}
& {[\mathbf{T c}(\mathbf{j} / \mathbf{i})]=} \\
& {\left[\begin{array}{cccccc}
\mathrm{a}_{1} & \mathrm{a}_{2} & \mathrm{a}_{3} & 0 & 0 & 0 \\
\mathrm{~b}_{1} & \mathrm{~b}_{2} & \mathrm{~b}_{3} & 0 & 0 & 0 \\
\mathrm{c}_{1} & \mathrm{c}_{2} & \mathrm{c}_{3} & 0 & 0 & 0 \\
\mathrm{c}_{1} y-\mathrm{b}_{1} z & \mathrm{c}_{2} y-\mathrm{b}_{2} z & \mathrm{c}_{3} y-\mathrm{b}_{3} z & \mathrm{a}_{1} & \mathrm{a}_{2} & \mathrm{a}_{3} \\
\mathrm{a}_{1} z-\mathrm{c}_{1} x & \mathrm{a}_{2} z-\mathrm{c}_{2} x & \mathrm{a}_{3} z-\mathrm{c}_{3} x & \mathrm{~b}_{1} & \mathrm{~b}_{2} & \mathrm{~b}_{3} \\
\mathrm{~b}_{1} x-\mathrm{a}_{1} y & \mathrm{~b}_{2} x-\mathrm{a}_{2} y & \mathrm{~b}_{3} x-\mathrm{a}_{3} y & \mathrm{c}_{1} & \mathrm{c}_{2} & \mathrm{c}_{3}
\end{array}\right]\left[\begin{array}{c}
\mathrm{Ru}_{\mathrm{ji}} \\
\mathrm{Rv}_{\mathrm{ji}} \\
\mathrm{Rw}_{\mathrm{ji}} \\
\mathrm{Tu}_{\mathrm{ji}} \\
\mathrm{Tv}_{\mathrm{ji}} \\
\mathrm{Tw}_{\mathrm{ji}}
\end{array}\right]}
\end{aligned}
$$

\section{Références}

[1] V. Stjskal, M. Vlasek, Kinematics and dynamics of machinery, 494 p., Marcell Dekker, Inc, New York, 1996

[2] P. Fanghella, Kinematics of spatial linkages by group algebrea, A structure based approch, Mechanism and machine theory 23(3) (1988) 171-183

[3] M. Fayet, P. Bonnet, Processus de détermination du rang des équations de liaisons, Distribution des mobilités, Mechanism and Machine Theory 30(2) (1995) 219-232

[4] G. Lagouin, Apport de la théorie des mécanismes à la conception des systèmes mécaniques assistée par ordinateur, Thèse, Université de Poitiers, France, 1992

[5] A. Mlika, Contribution à la systématisation de l'analyse cinématique et statique des mécanismes, Thèse de Doctorat, ENIT, Université de Tunis, Juillet 1999

[6] P. Deplanche, A. Leroy, Étude cinématique systématique des mécanismes parfaits, Technologie et formations $\mathrm{n}^{\circ} 42$ (1992) 33-42

[7] F. Harary, H. Yan, Logical foundations of kinematic chains : graphs, line graphs, and hypergraph, J. mechanical design 112 (March 1990) 79-83

[8] P. Fanghella, C. Galletti, On the choice of independant loops in mechanism, Computational kinematics, J.-P. Merlet, B. Bravani (ed.), 1995, pp. 113-122

[9] M. Gondran, M. Minoux, Graphes et algorithmes, 545 p., $2^{\mathrm{e}}$ édition, Eyrolles, 1990

[10] A. Kauffman, Méthodes et modèles de la recherche opérationnelle. Tome 2, 544 p., $2^{\text {ème }}$ édition, Dunod 1968

[11] S. Even, Graph algorithms, 249 p., Computer science press, Inc. USA, 1979

[12] C. Bohatier, A numerical method for mechanisms equations automatic building, European J. mechanics, A/Solids 9(4) (1990) 359-371

[13] R. Aviles, A. Hernandez, I. Liorente, Analysis and optimum sunthesis of mechanisms based on the finite element method, Actes du congrès international Strucome 88, Novembre 1988, pp. 671-684

[14] L. Romdhane, A dyad search algorithm for solving planar linkages using the dyad method, Les annales maghrébines de l'ingénieur, vol. 9(1), Avril 1995 